\title{
Sequential Beamforming Synthetic Aperture Imaging
}

\author{
Kortbek, Jacob; Jensen, Jørgen Arendt; Gammelmark, Kim Løkke
}

Published in:

Ultrasonics

Link to article, DOI:

10.1016/j.ultras.2012.06.006

Publication date:

2013

Document Version

Publisher's PDF, also known as Version of record

Link back to DTU Orbit

Citation (APA):

Kortbek, J., Jensen, J. A., \& Gammelmark, K. L. (2013). Sequential Beamforming Synthetic Aperture Imaging. Ultrasonics, 53(1), 1-16. https://doi.org/10.1016/j.ultras.2012.06.006

\section{General rights}

Copyright and moral rights for the publications made accessible in the public portal are retained by the authors and/or other copyright owners and it is a condition of accessing publications that users recognise and abide by the legal requirements associated with these rights.

- Users may download and print one copy of any publication from the public portal for the purpose of private study or research.

- You may not further distribute the material or use it for any profit-making activity or commercial gain

- You may freely distribute the URL identifying the publication in the public portal

If you believe that this document breaches copyright please contact us providing details, and we will remove access to the work immediately and investigate your claim 


\title{
Sequential beamforming for synthetic aperture imaging
}

\author{
Jacob Kortbek $^{\mathrm{a}, \mathrm{b}, *}$, Jørgen Arendt Jensen ${ }^{\mathrm{b}}$, Kim Løkke Gammelmark ${ }^{\mathrm{a}}$ \\ a BK Medical, 2730 Herlev, Denmark \\ ${ }^{\mathrm{b}}$ Department of Electrical Engineering, Center for Fast Ultrasound, Technical University of Denmark, Denmark
}

\section{A R T I C L E I N F O}

\section{Article history:}

Received 6 December 2010

Received in revised form 2 March 2012

Accepted 1 June 2012

Available online 28 June 2012

\section{Keywords:}

Beamforming

Synthetic aperture

Ultrasound imaging

\begin{abstract}
A B S T R A C T
Synthetic aperture sequential beamforming (SASB) is a novel technique which allows to implement synthetic aperture beamforming on a system with a restricted complexity, and without storing RF-data. The objective is to improve lateral resolution and obtain a more depth independent resolution compared to conventional ultrasound imaging. SASB is a two-stage procedure using two separate beamformers. The initial step is to construct and store a set of B-mode image lines using a single focal point in both transmit and receive. The focal points are considered virtual sources and virtual receivers making up a virtual array. The second stage applies the focused image lines from the first stage as input data, and take advantage of the virtual array in the delay and sum beamforming. The size of the virtual array is dynamically expanded and the image is dynamically focused in both transmit and receive and a range independent lateral resolution is obtained. The SASB method has been investigated using simulations in Field II and by off-line processing of data acquired with a commercial scanner. The lateral resolution increases with a decreasing $F \#$. Grating lobes appear if $F \# \leqslant 2$ for a linear array with $\lambda$-pitch. The performance of SASB with the virtual source at $20 \mathrm{~mm}$ and $F \#=1.5$ is compared with conventional dynamic receive focusing (DRF). The axial resolution is the same for the two methods. For the lateral resolution there is improvement in FWHM of at least a factor of 2 and the improvement at $-40 \mathrm{~dB}$ is at least a factor of 3 . With SASB the resolution is almost constant throughout the range. For DRF the FWHM increases almost linearly with range and the resolution at $-40 \mathrm{~dB}$ is fluctuating with range. The theoretical potential improvement in SNR of SASB over DRF has been estimated. An improvement is attained at the entire range, and at a depth of $80 \mathrm{~mm}$ the improvement is $8 \mathrm{~dB}$.
\end{abstract}

(c) 2012 Elsevier B.V. All rights reserved.

\section{Contents}

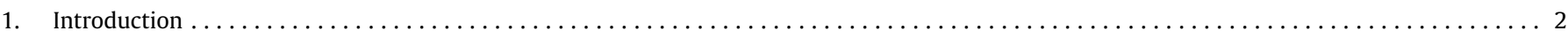

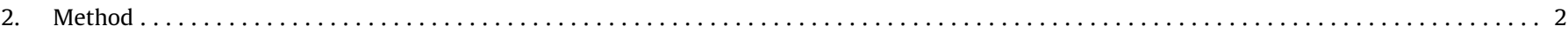

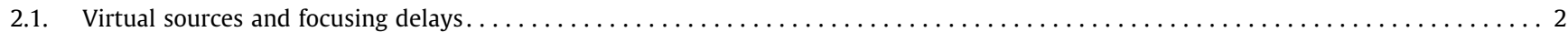

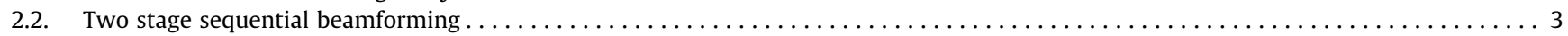

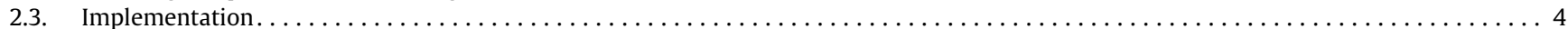

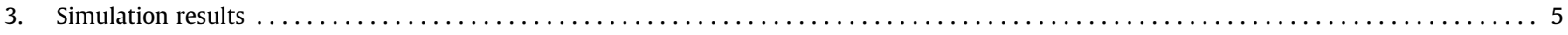

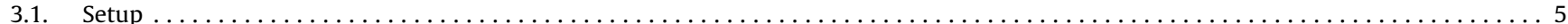

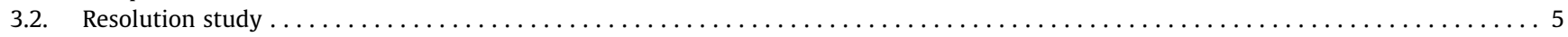

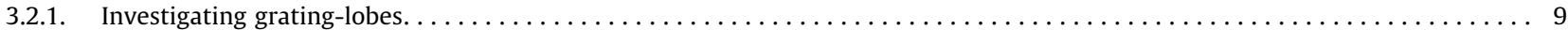

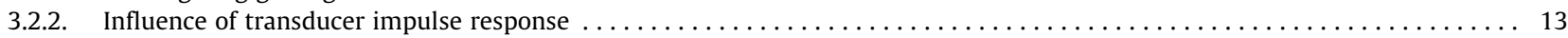

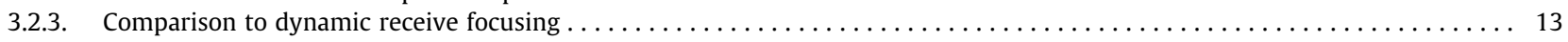

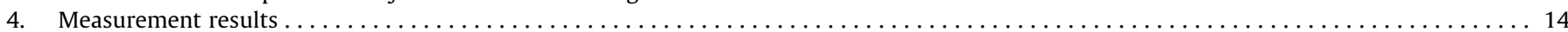

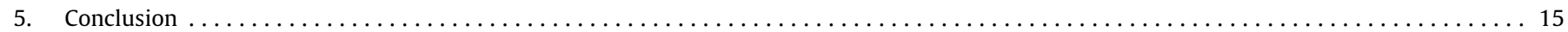

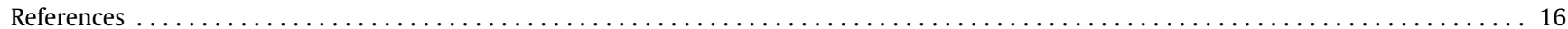

\footnotetext{
* Corresponding author at: BK Medical, 2730 Herlev, Denmark.

E-mail address: jbk@bkmed.dk (J. Kortbek).
} 


\section{Introduction}

Inspired by synthetic aperture (SA) radar techniques [1-3], SA ultrasound imaging has been investigated thoroughly for many years. In synthetic transmit aperture (STA) imaging [4,5] a single element is used to transmit a spherical wave that occupies the entire region of interest. The backscattered signals are registered using a multi-element receive aperture and RF-samples from all channels are stored. Delay-and-sum (DAS) beamforming can be applied to these data to construct a low-resolution image (LRI), hence an image constructed from a single emission. Several emissions from single elements across the aperture will synthesize a larger aperture and the LRI's from these emissions can be added into a single high-resolution image (HRI). The HRI is dynamically focused in both transmit and receive yielding an improvement in resolution [6]. This has been confirmed with side-by-side comparisons with conventional ultrasound imaging in pre-clinical trials by Pedersen et al. [7]. This imaging technique sets high demands on processing capabilities, data transport, and storage and makes implementation of a full SA system very challenging and costly. The method investigated in this paper is another variation on SA processing. The objective is to reduce the demands on the system making it a more realistic task to implement, while still preserving benefits in imaging performance.

Many variations of synthetic aperture focusing (SAF) and examples of implementation have been reported with improvements in both frame rate, penetration, and lateral resolution. A simple realtime single channel system with a multiplexer for array imaging was described by Peterson and Kino [8] back in 1984. In the mono-static approach [6] the same element serves as a transmitter and a receiver. A SA technique suitable for a hand held system using a multi-element transmit and receive aperture was described by Karaman et al. [4]. Lockwood and Hazard described a sparse SA beamforming technique for three-dimensional ultrasound imaging using a few transmit pulses for each image [9,10]. A SA method for a circular aperture was investigated by O'Donnell and Thomas [11].

The concept of using the transmit focal point as a virtual source (VS) or a virtual aperture was introduced by Passmann and Ermert [12]. Virtual sources in SAF was further investigated by Frazier and O'Brien [13], Nikolov and Jensen [14,15], and Bae and Jeong [16]. It was shown that the virtual source coincides with the focal point of the transducer, and that a depth independent resolution can be achieved.

Mono-static SA focusing applied to imaging with a single rotating mechanically focused concave element was investigated by Kortbek et al. [17]. Such an imaging system can e.g. be found in an anorectal ultrasound transducer. One objective of this study was to improve lateral resolution. This was done by storing the RF-lines (the beamformed image-lines before envelope detection) from every emission and feeding these to a SA beamformer. Due to the small radius of rotation, the synthesized aperture only experiences a moderate lateral expansion, and the improvement in lateral resolution was not significant.

In this paper the same SAF technique is applied but to linear array imaging. Here the synthesized aperture becomes considerable larger with a lateral translation of the VS than with a rotation. Instead of having a VS created from a single concave element physically focused in both transmit and receive, the VS is now created from a sub-aperture - a group of elements. This offers more flexibility, since the position and the opening angle of the VS are determined by the electronic focusing instead of the physical concave shape of the single element transducer. The receive focusing is a simple fixed focusing with the receive focal point at the same position as the transmit focal point. This first beamformer, thus, mimics the focusing that is a consequence of the concave single element transducer. The focused RF-lines from every emission are stored and transferred to a SA beamformer just as the one applied with the rotating transducer. Two beamformers are, thus, applied sequentially - a simple fixed focus beamformer and a SA beamformer, and the method is denoted Synthetic Aperture Sequential Beamforming (SASB).

One objective of SASB is to improve the penetration depth. The primary objective though is to improve lateral resolution and obtain a more range independent resolution compared to conventional ultrasound imaging. Contrary to a full SA setup only a single RF-line is beamformed and stored for each emission. This reduces the system requirements significantly. For simplicity the method is investigated using a linear array, but it might as well apply to other types of imaging. The investigation comprises performance evaluation at a large imaging range. This is done without consideration for the typical use of the specific transducer but as a mean to demonstrate the properties of the method.

The SASB method is described in more detail in Section 2. Section 3 presents results from the Field II $[18,19]$ simulation study. A parameter study shows the performance and artifacts of the method and compare with conventional dynamic receive focusing (DRF). Section 4 presents imaging examples and signal-to-noise ratio (SNR) calculations with data acquired from phantom measurements with a commercial scanner.

\section{Method}

Synthetic aperture sequential beamforming (SASB) is a twostage procedure which can be applied to B-mode imaging with any array transducer. The initial step is to construct and store a set of B-mode image lines using a conventional sliding sub-aperture. These 1 st stage lines are obtained with a single focal point in both transmit and receive. The second stage consists of an additional beamformer using the focused image-lines from the first stage as input data. The concept of virtual sources and means of calculating the focusing delays are briefly discussed in Section 2.1 before presenting the SASB in more detail in Section 2.2.

\subsection{Virtual sources and focusing delays}

In delay-and-sum receive focusing appropriate delays are applied to the responses of the individual transducer elements originating from the focusing point and coherently adding these responses. The delays are found from the round trip time-of-flight (TOF), which is the propagation time of the emitted wave in its path from the transmit origin, $\vec{r}_{e}$ to the focusing point (FP), $\vec{r}_{f p}$ and return to one of the elements of the receive aperture, $\vec{r}_{r}$ as illustrated in Fig. 1. This could be the case of mono-static SAF.

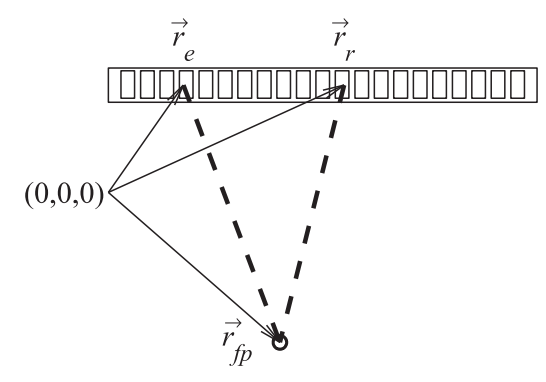

Fig. 1. Wave propagation path (dotted line) for calculating the time-of-flight in receive focusing. The transmit origin, $\vec{r}_{e}$ and the receive point, $\vec{r}_{r}$ are illustrated as different elements of an array. 
$t_{t o f}=\frac{d}{c}=\frac{\left|\vec{r}_{f p}-\vec{r}_{e}\right|+\left|\vec{r}_{r}-\vec{r}_{f p}\right|}{c}$.

$d$ is the length of the path, and $c$ is the speed of sound. The sample index of the temporal RF signal is calculated by simply multiplying $t_{\text {tof }}$ with the sampling frequency, $f_{s}$. The transmit origin is not necessarily well defined, if the emitted pressure wave does not emanate from a single element point source as illustrated in Fig. 1. In multi-element synthetic aperture imaging [4] a multi-element transmit sub-aperture is introduced as an alternative to mono-static SAF to ensure sufficient transmitted energy. Here the pressure wave is the result of numerous waves emitted from multiple elements. With a focused transmission the focal point is introduced as a virtual source, since a spherical wave emanates from this point in a limited angular region. With a known position of the source this allows for the SAF delays to be calculated.

Applying a VS instead of a single element source has consequences for the calculations of SA focusing delays. Time-delay calculations for different applications was discussed by Kortbek et al. [20]. In dynamic receive beamforming a new set of delay values is calculated for each focusing point (FP). Assuming the speed of sound $c$ is known, the delay value, $t_{d}$ for the receiving element with position $\vec{r}_{r}$ is calculated in accordance with Fig. 2 (left)

$t_{d_{f p}}\left(\vec{r}_{r}\right)=\frac{\left|\vec{r}_{t f p}-\vec{r}_{e}\right| \pm\left|\vec{r}_{f p}-\vec{r}_{t f p}\right|}{c}+\frac{\left|\vec{r}_{r}-\vec{r}_{f p}\right|}{c}$

The transmit origin, $\vec{r}_{e}$ is the center element of the transmit subaperture. $\vec{r}_{t f p}$ and $\vec{r}_{f p}$ are the positions of the transmit focal point and the receive focusing point respectively. The \pm in (2) refer to whether the FP is above or below the VS. With SASB the first stage beamformer has a fixed receive focus and the delay values, $t_{d}$ are calculated in accordance with Fig. 2 (right)

$$
\begin{aligned}
t_{d_{f p}}\left(z_{v}\right) & =\frac{1}{c}\left(\left|\vec{r}_{t f p}-\vec{r}_{e}\right| \pm\left|\vec{r}_{f p}-\vec{r}_{t f p}\right| \pm\left|\vec{r}_{t f p}-\vec{r}_{f p}\right|+\left|\vec{r}_{r}-\vec{r}_{t f p}\right|\right) \\
& =\frac{1}{c}\left(2 z_{v} \pm 2\left|\vec{r}_{t f p}-\vec{r}_{f p}\right|\right)
\end{aligned}
$$

where $z_{v}$ is the distance from the aperture to the VS. With dynamic receive beamforming the differences between the individual channel delays changes with the position of the focusing point due to the term $\left|\vec{r}_{r}-\vec{r}_{f p}\right|$ in (2). A new set of delay values are, thus, calculated for each focusing point. With fixed receive focusing as in (3) only a single set of delay values is calculated and for each focusing point a constant is added. This is an important issue in respect to the complexity of the beamformer. With dynamic focusing the delay calculations involve among others the computational costly square root operation.
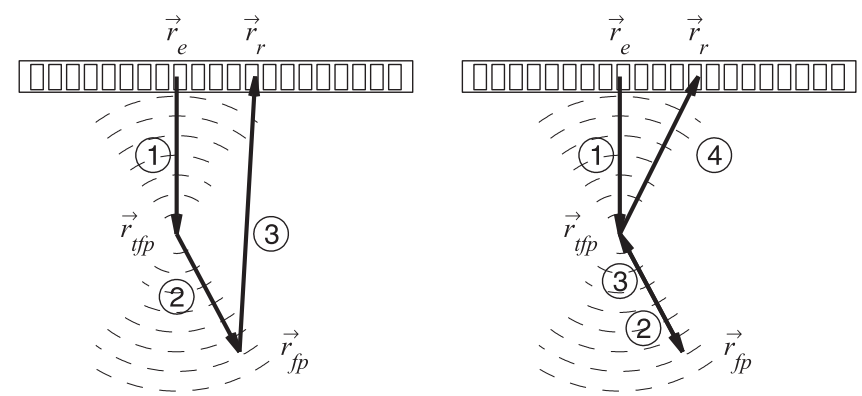

Fig. 2. Wave propagation path (solid line) for calculating the receive focusing time delays for a focused transmission. The center element of the transmitting aperture is perceived as the transmit origin. Dynamic receive focusing (left) and fixed receive focusing (right).

\subsection{Two stage sequential beamforming}

The objective of the proposed method is to obtain a synthetic transmit focus using data from multiple emissions but without storing the channel data. This is achieved in a two stage process. The 1st stage RF-lines, which are the output from the first beamformer, are obtained with a single focal point in both transmit and receive. This focal point is considered as a VS emitting a spherical wave front spatially confined by the opening angle. Each point in the 1st stage focused image line contains information from the arc of a circle that crosses that point. The arc has center in the focal point and is limited by the opening angle. This is illustrated in Fig. 3. Here it is also possible to see that the highlighted image point at $x=0$ is represented in a phase-exact manner in multiple 1 st stage lines obtained from multiple emissions. This means that the channel delay profile for focusing the highlighted image point using channel data from the first emission is exactly the same as the profile used for creating the highlighted point on the 1st stage line from the first emission. The profiles are the same since the image point is on the arc intersecting the point in the 1st stage line. This is also supported by the illustration in Fig. 2 (right). This characteristic is a consequence of the fixed receive focus and is exploited in the second stage. Here all the representations from different emissions of an image point (in form of samples from different 1st stage lines) are coherently added. A synthetic transmit focus is thus obtained without storing channel data. If the 1st stage is exchanged with a dynamically focused beamformer an image point is no longer exactly presented in any points of any 1 st stage lines. The channel delay profile is unique for every point if dynamic focus is applied, which is also illustrated in Fig. 2 (left). Another consequence of having a dynamically focused beamformer in the 1st stage instead of a fixed focus beamformer is a large increase in implementation complexity which is discussed further in Section 2.3.

Each HRI consists of a number of high resolution image lines the output lines from the 2nd stage beamformer. In this paper, $N$ denotes the number of emissions, which is the same as the number of 1 st stage lines and also the number of high resolution lines. The method has in principle no restrictions toward these numbers. Each sample in a high resolution line (HRL) is constructed in the second beamformer by selecting a sample from each of those $1 \mathrm{st}$ stage lines, which contain information from the spatial position of the image point and summing a weighted set of these samples.

The number of elements in the transmit sub-aperture, the depth of the focal point and, thus, the F\# determines the opening angle,

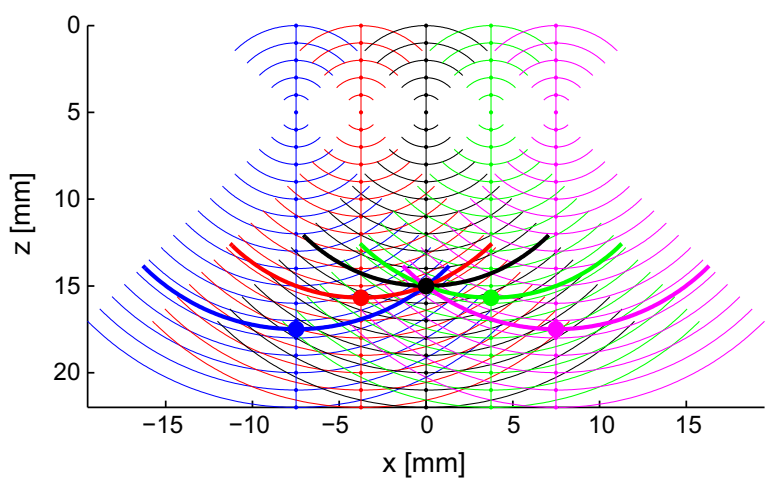

Fig. 3. Example of wave propagation and 1st stage image lines from 5 different emissions. Each point on the image lines contains information from the spatial positions which are indicated by the arc of a circle intersecting the point. A single high resolution image point of the SASB method is obtained by extracting information from all of those 1st stage image lines which contain information about that point. 


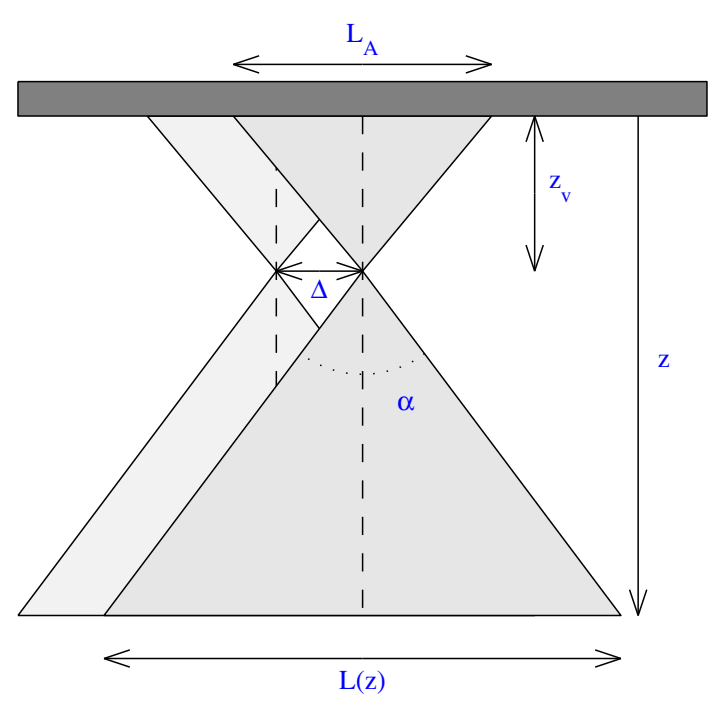

Fig. 4. Geometry model of the emitted wave fields from two consecutive emissions. The lateral width, $L(z)$ of the wave field at a depth, $z$ determines the number of LRL's which can be added in the 2nd stage beamformer for an image point at depth, $z$.

hence the width of the transmitted wave field and the point spread function (PSF). As a consequence of the spatially limited wave propagation it must be determined for each image point in a HRL which emissions that have a wave field encompassing the image point. These emissions contribute to the HRL, and samples from each of them are selected according to the SAF delays, and added together. By definition a single HRL is composed of the sum of a number of low resolution lines (LRLs). A single LRL is, thus, the contribution from a given emission to a HRL.

The SAF can be formulated as a sum over LRL's. A single sample of the HRL, representing the image point at the location $\vec{r}_{i p}$ with lateral coordinate $x$ and axial coordinate $z$, can be expressed as

$h(x, z)=\sum_{k=1}^{K(z)} \mathcal{W}\left(x_{k}, z\right) l_{x_{k}}(z)$.

$h(x, z)$ is the HRL sample, and $l_{x_{k}}(z)$ is the LRL sample from the emission with lateral position $x_{k}$. The variable $\mathcal{W}$ is an apodization function with $K(z)$ values, which controls the weighting of the contribution from each of the LRL's. It is a function of the axial position of the image point, since the number of contributing emissions, $K(z)$ increases with the distance to the VS. $K$ determines the number of VS's applied for a given HRI point and is a measure of the size of the synthesized aperture. $K(z)$ can be calculated directly from the geometry shown in Fig. 4 as

$K(z)=\frac{L(z)}{\Delta}=\frac{2\left(\left|z-z_{v}\right|\right) \tan (\alpha / 2)}{\Delta}$

showing that $K$ increases linearly with the distance to the virtual source. This facilitates a more range independent lateral resolution compared to DRF since contrary to DRF transmit focusing is also dynamic with the dynamic expansion of the size of the synthezized aperture, $K . L(z)$ is the lateral width of the wave field at a depth, $z$, and $\Delta$ is the distance between the VS's of two consecutive emissions. $\alpha$ is the opening angle of the VS and is the angular span for which the phase of the wave field can be considered constant. The opening angle can be expressed as

$\alpha=2 \arctan \frac{1}{2 F \#}$,

which is a valid approximation [21]. With $L_{A}$ denoting the size of the sub-aperture the $F$-number becomes $F \#=z_{v} / L_{A}$. The LRL can be formulated using the RF-line, $s_{x_{k}}\left(z^{\prime}\right)$ $l_{x_{k}}(z)=s_{x_{k}}\left(z^{\prime}\right)$

$z^{\prime}$ is the axial position at which to select a sample from the RF-line. $z^{\prime}$ can be found from the distance function, $z^{\prime}=d\left(\vec{r}_{v_{x_{k}}}, \vec{r}_{i p}\right) / 2$ which calculates the transmit-receive round trip travel path for the SAF, and thus the sample index for the RF-line, $s_{x_{k}}\left(z^{\prime}\right)$. The transducer elements are electronically focused at the VS at the position $\vec{r}_{v_{x_{k}}}$ with a focal distance of $z_{v}$ from the aperture. The aperture is focused in both transmit and receive and the distance function becomes a sum of transmit and receive travel paths

$d\left(\vec{r}_{v_{x_{k}}}, \vec{r}_{i p}\right)=2 z_{v} \pm 2\left|\vec{r}_{i p}-\vec{r}_{v_{x_{k}}}\right|$

The \pm in (8) refer to whether the image point is above or below the VS. A single sample of the HRL can thus be formulated using (4) and (7)

$h(x, z)=\sum_{k=1}^{K(z)} \mathcal{W}\left(x_{k}, r\right) s_{x_{k}}\left(d\left(\vec{r}_{v_{x_{k}}}, \vec{r}_{i p}\right)\right)$.

The formulation of the method in this section assumes an aperture with an infinite number of elements. This becomes apparent when observing (5). At greater depth $K(z)$ will exceed the number of available 1st stage lines, $N$. At depths beyond the point where $K(z)=N$ the synthesized aperture will no longer increase with depth. The $F \#$ will increase and the lateral resolution will no longer be range independent beyond that depth. Here it is also worth mentioning that as $K(z)$ increases the difference in acquisition time between the 1st stage lines applied increases and motion artifacts might become an issue. The synthesized aperture will also decrease for the HRL's near the edges, compared to the center HRL because of the limited number of 1 st stage lines available. The lateral resolution is, thus, laterally dependent. The apodization function $\mathcal{W}$ is also laterally dependent and asymmetric.

The HRI is composed of a sum of LRI's from multiple transmissions as formulated in (9). The formulation assumes that the image object is stationary during all transmission, which is not the case in vivo. Tissue motion and motion artifacts are nevertheless not completely destructive to SA imaging. The susceptibility to motion of SA imaging has been investigated by several authors [22-30], and techniques to address the problems with tissue motion have been demonstrated.

\subsection{Implementation}

In SAF data from multiple emissions are applied. This could essentially decrease the image frame rate if a full set of emissions is needed to construct and update the HRI. Nikolov and Jensen proposed SA recursive imaging [31] to be able to construct a new frame after each emission. In SASB acquisition of a full set of $N$ emissions is a necessity before the first HRI can be constructed, but by storing the $M$ most recent 1 st stage lines a new HRL can be constructed after each emission using the already stored lines and the newly acquired line. This is feasible by having a temporal offset between the reconstructed HRL and the acquired RF-line corresponding to $M$ emissions. For HRI number $i H R I_{i}$ the HRL's are numbered $J=1,2, \ldots N$. The corresponding RF-lines across the aperture are denoted $j=1,2, \ldots N$, and for $H R I_{i+1}$ they are denoted $j=N+1, N+2, \ldots 2 N$. The HRL $J$ of $H R I_{k}$ can be constructed while acquiring the RF-line, $j=J+M$. With $M$ being equal to the number of available channels of the 2nd stage beamformer the temporal offset is minimized. Each 1st stage line makes a contribution to several HRL in the 2nd stage beamformer. All of these HRL can in principle be refreshed after each acquisition. This is solely determined by the capability of the 2nd stage beamformer to do parallel beamforming. 
A view on implementation of the SASB at a block function level demonstrates a requirement for two beamformers and memory for storage of the 1st stage image lines to feed the 2nd stage beamformer. The 1st stage beamformer is simple since the receive delay-profiles are the same for all image points except for a depth dependent additive constant. It only requires calculation of a single profile or a look up table (LUT) with a single entry. It could easily be analog and thereby save many ADC's. The 2 nd stage beamformer has the complexity of a general dynamic receive focusing beamformer. For each depth a separate entry in a LUT for a profile is required or the delay profile must be calculated. Apodization is also a desirable requirement for the 2 nd stage beamformer to suppress off-axis energy lobes. Since $K(z)$ increases linearly with depth the apodization is dynamic. The apodization is also a function of the lateral position of the HRL due to the finite aperture and it can profitably be calculated as a parametric function. The desired number of channels of a single-beam beamformer in the 2nd stage equals the number of acquired 1st stage lines to synthesize the full size array. The channel count, $C$ of the 2 nd stage beamformer can be reduced though with a factor of $M$ to $C=N / M$ using a $M$-beam beamformer capable of processing $M$ parallel beams and an additional accumulation buffer for temporary output lines. In this form and in a case of $N 1$ st stage lines ( $N$ acquisitions) and $N$ 2nd stage lines the 2nd stage temporary output lines of the beamformer are $L_{\text {out }}=\bmod ([0:(M-1)] C+n-1, N)+1$ using the 1 st stage lines for the input of the beamformer $L_{\text {in }}=\bmod ([0:(C-1)]+n-1, N)+1$ for beamforming operation $n$. After $N$ operations each output line has been generated $M$ times using $M$ different sets of input signals (different virtual sources). By accumulating the $M$ temporary output lines in the buffer the resulting output lines are exactly the same as if a single-beam $N$-channel beamformer is used.

\section{Simulation results}

In this section the method is investigated using simulations in Field II $[18,19]$ and SAF is carried out using the Beamformation Toolbox, BFT2 [20]. Images of point targets using different realizations of SASB are created. The axial and lateral resolution are extracted and compared to conventional B-mode imaging. The simulations are made with a model of a linear array $7 \mathrm{MHz}$ transducer with properties similar to a commercial transducer.

\subsection{Setup}

The parameters, which are the most decisive for the performance of SASB are the focal depth $z_{v}$ (the focal depth), and the $F \#$ of the VS. A parameter study is done by varying $z_{v}$ with values of $5,10,15,20 \mathrm{~mm}$, and the $F \#$ with values in the range $0.5-2.5$. The study is done with a default set of transducer parameters and processing parameters shown in Table 1.

In the main parameter study the value of $N_{e}=401$ has been set to exclude the effects of a finite aperture to demonstrate the ability of the method to generate a range independent resolution. The number of 1 st stage image lines and also 2 nd stage image lines is the same as the number of transducer elements.

In this study a single cycle sinusoid is used as excitation and a weighted double cycle sinusoid is used to simulate the transducer impulse response. Another less extensive study is performed with more practical realizable parameters. The number of elements is $N=191$. A double cycle sinusoid forms the excitation, and the measured impulse response of the applied transducer type is used. The scattering media consists of stationary point targets (PTs) placed in the center of the image in the range from $5 \mathrm{~mm}$ to $95 \mathrm{~mm}$ with a distance of $10 \mathrm{~mm}$.
Table 1

Default values for the simulation parameter study.

\begin{tabular}{lll}
\hline Parameter & & Value \\
\hline Various & $f_{s}$ & \\
Sampling frequency & & $120 \mathrm{MHz}$ \\
Transducer & $\Delta$ & \\
Pitch & $f_{c}$ & $0.208 \mathrm{~mm}$ \\
Center frequency & $B$ & $7 \mathrm{MHz}$ \\
Bandwidth, relative & $z_{e l e}$ & 0.6 \\
Elevation focus & $N$ & $25 \mathrm{~mm}$ \\
Number of elements & & 401 \\
Excitation & & 1 Cycle sinusoid \\
1st Stage processing & & \\
Focusing & $N_{1 s t}$ & Fixed, (xmt/rcv) \\
Number of channels, xmt/rcv & & $63 / 63$ \\
Transmit sub-aperture & $\mathcal{A}_{x m t}$ & Symmetric only \\
Transmit apodization & $z_{v}$ & Hamming \\
Focal depth (virtual source) & $\mathcal{A}_{r c v}$ & $5,10,15,20 \mathrm{~mm}$ \\
Receive apodization & & Hamming \\
Receive sub-aperture & $N$ & Same as transmit \\
Number of image lines & $\Delta$ & 401 \\
Distance between lines & & $0.208 \mathrm{~mm}$ \\
2nd Stage processing & & \\
Focusing & & Synthetic aperture \\
Number of channels & $N_{2 n d}$ & 401 \\
SA weighting & $\mathcal{W}$ & Hamming \\
Number of image lines & $N$ & 401 \\
Distance between lines & & $0.208 \mathrm{~mm}$ \\
Applied 1st stage lines & & Symmetric only \\
& &
\end{tabular}

\subsection{Resolution study}

Different realizations of SASB are simulated to evaluate performance and limitations. The position of the VS and the opening angle determines the image area covered by a single emission. If the covered area increases, the number of 1 st stage lines, which can be used for the 2nd stage beamforming, also increases, yielding a larger synthesized aperture. This explicitly sets demands on the size of the array and the number of required 2nd stage beamformer channels if a range independent resolution is the objective. The study will show that other complications exist with the combination of a shallow focal depth and a large opening angle.

The log compressed envelope HRI's are shown in this section. The axial and lateral resolution are extracted at the image center for several depths. The resolution is quantified at $-6 \mathrm{~dB}$, the Full Width at Half Maximum (FWHM), and at $-40 \mathrm{~dB}$. For each PT the
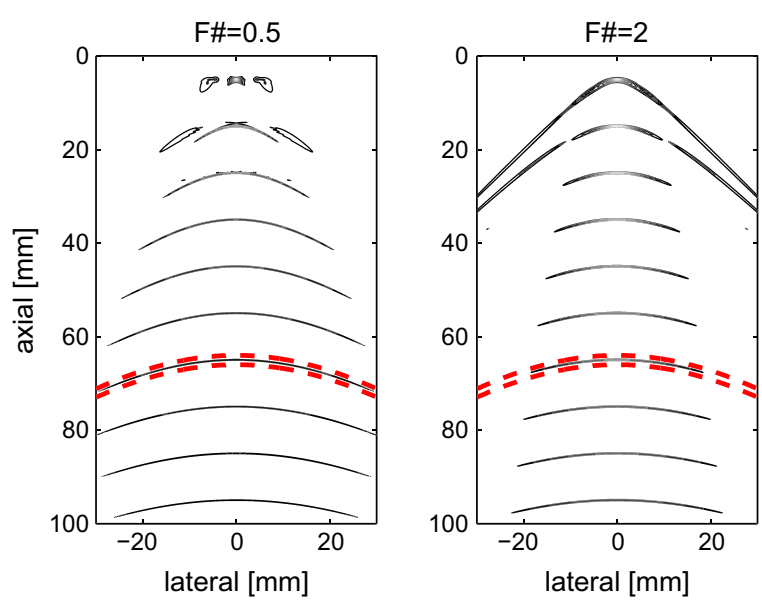

Fig. 5. Contour plots of the envelope of the output from the 1st stage beamformer $F \#=0.5$ (left), and $F \#=2$ (right). The VS is at $5 \mathrm{~mm}$. The curvature of the PSF is determined by the distance between the VS's and the point of the PSF. This is calculated analytically and shown as dotted lines for two points just above and below the PT at $z=65 \mathrm{~mm}$. 

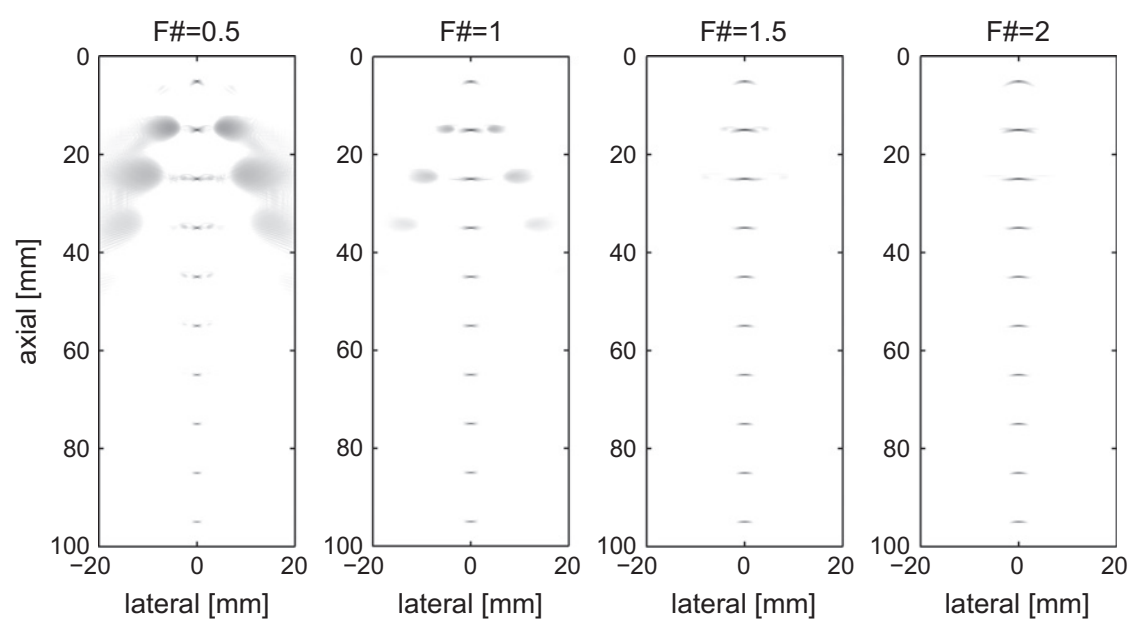

Fig. 6. SASB Images with the VS at $5 \mathrm{~mm}$ and with different values of $F \#$. Dynamic Range is $60 \mathrm{~dB}$.
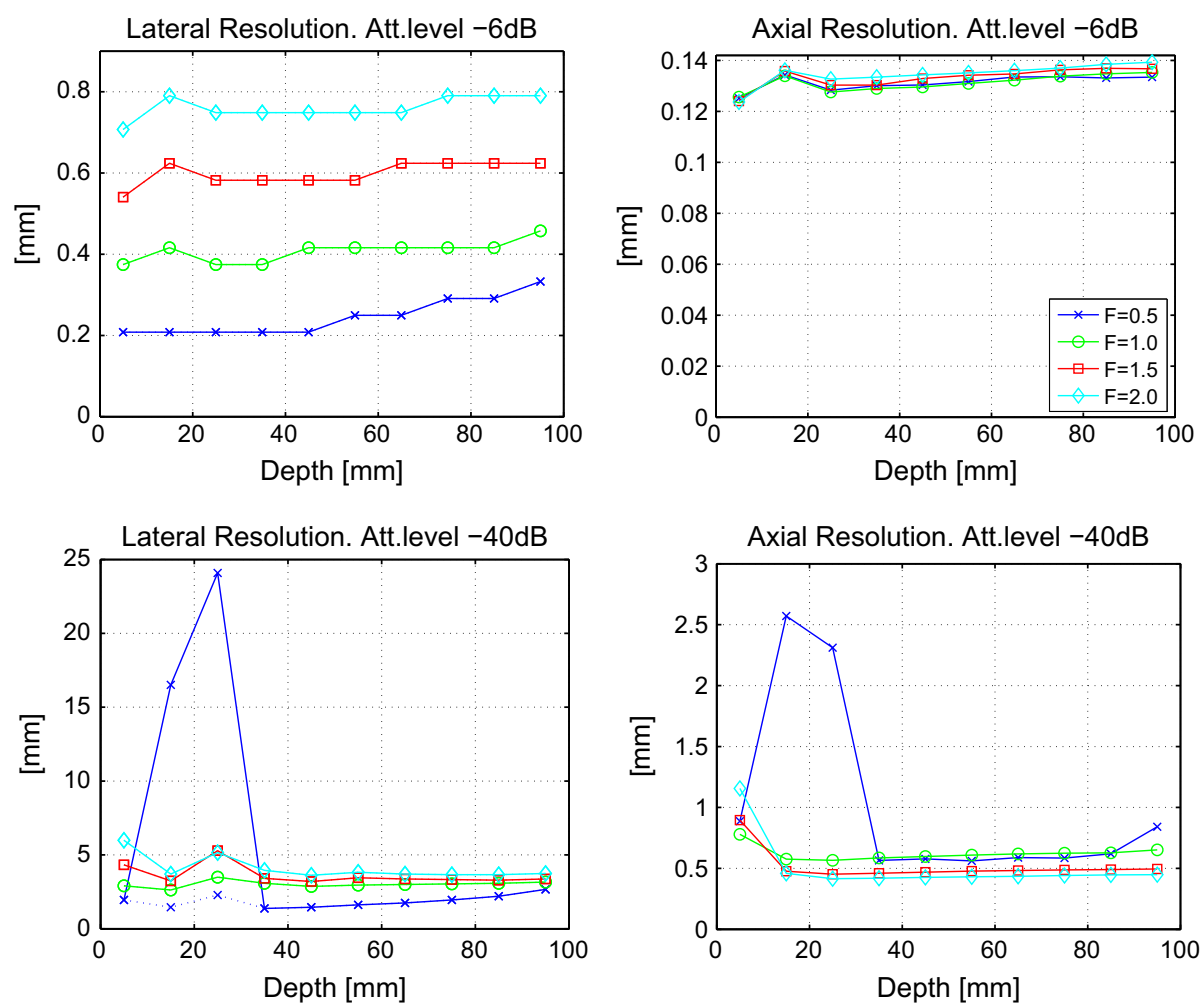

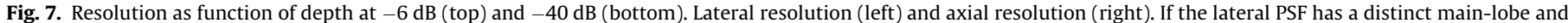
side-lobe distribution, the main-lobe resolution is shown as a dotted line. Shown for the four different setups represented in Fig. 6 .

resolution is calculated as the width at which the envelope amplitude has decreased to the specified level relative to the maximum level at that given PT. For the lateral resolution the envelope amplitude is taken as the maximum amplitude within a axial range of $\pm 3 \mathrm{~mm}$ around the PT. Likewise for the axial resolution the envelope amplitude is taken as the maximum amplitude across the entire lateral range of the image.

A poor lateral resolution is the consequence of having a PSF with a wide main lobe and/or high side-lobe levels. The illustrations of the quantified lateral resolution reflects this by also showing the main-lobe resolution as a dotted line, but only if the lateral PSF has a distinct main-lobe and side-lobe distribution. That is if the lateral PSF drops below the $-40 \mathrm{~dB}$ level and rises above this level again at a greater lateral position.

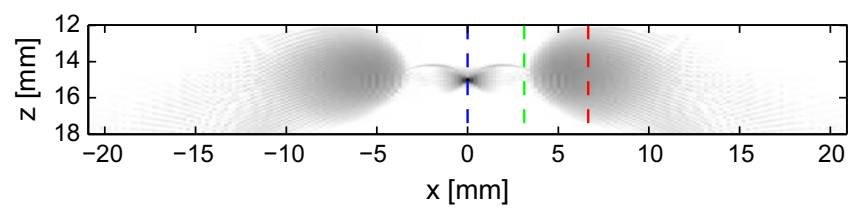

Fig. 8. A zoom in on the HRI shown in Fig. 6 for $F \#=0.5$. Three different HRL's are marked with dotted lines. The LRL's composing these HRL's are shown in Figs. 9-11. 
LRL

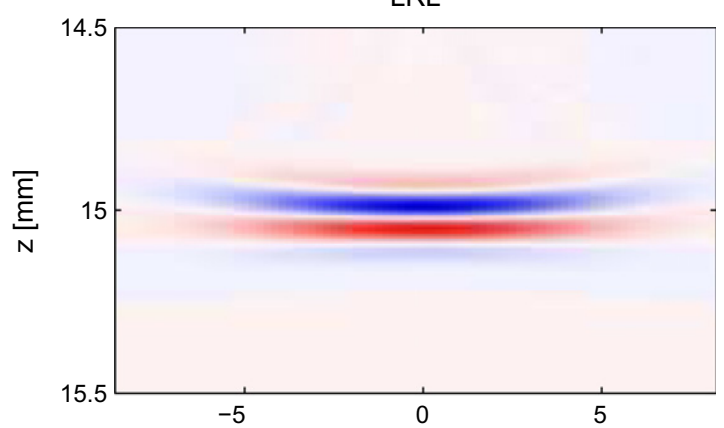

Weighted LRL (WLRL)

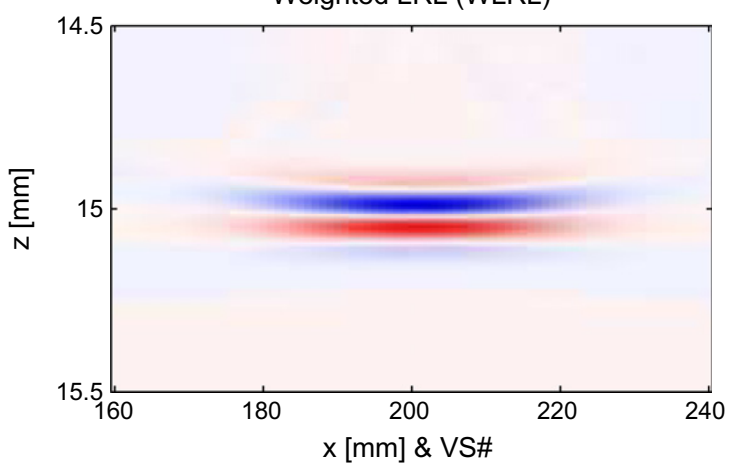

Norm. $\Sigma$ LRL

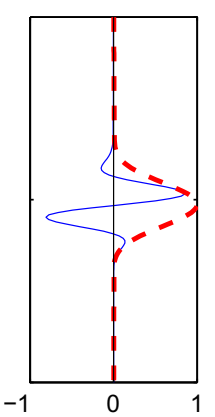

Norm. $\Sigma$ WLRL

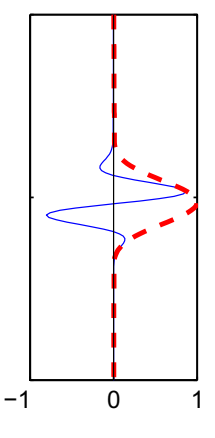

Env. Rel. to HRI

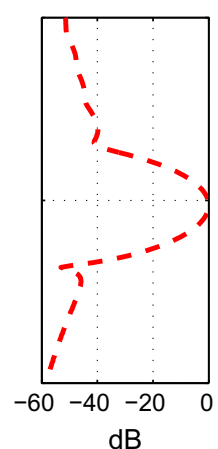

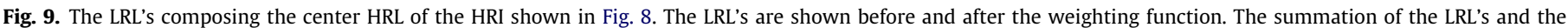
corresponding envelope are also shown.
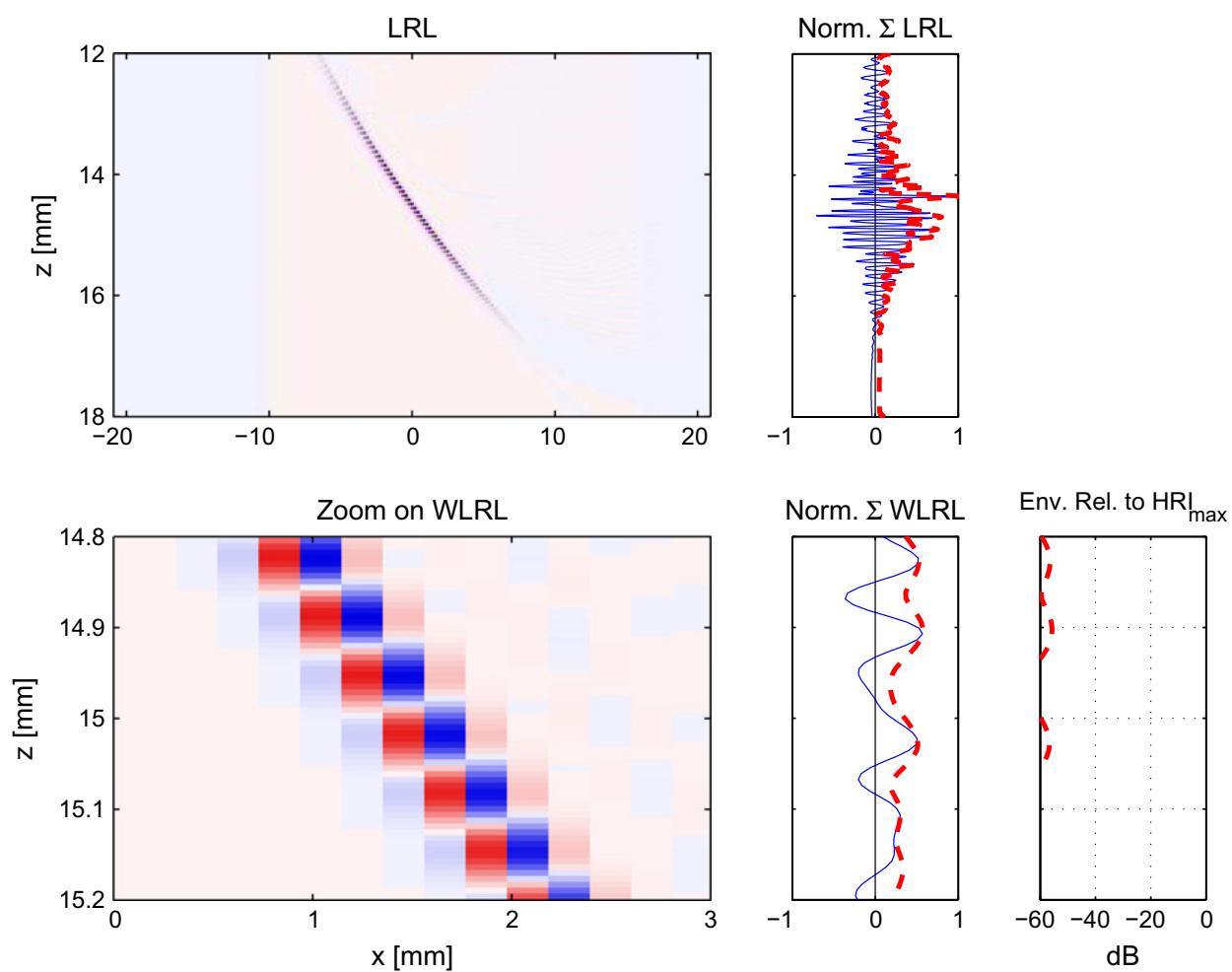

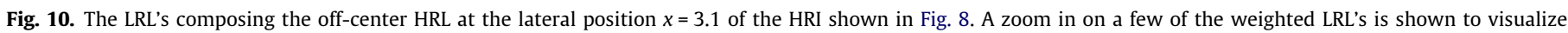
destructive summation of the LRL's. 

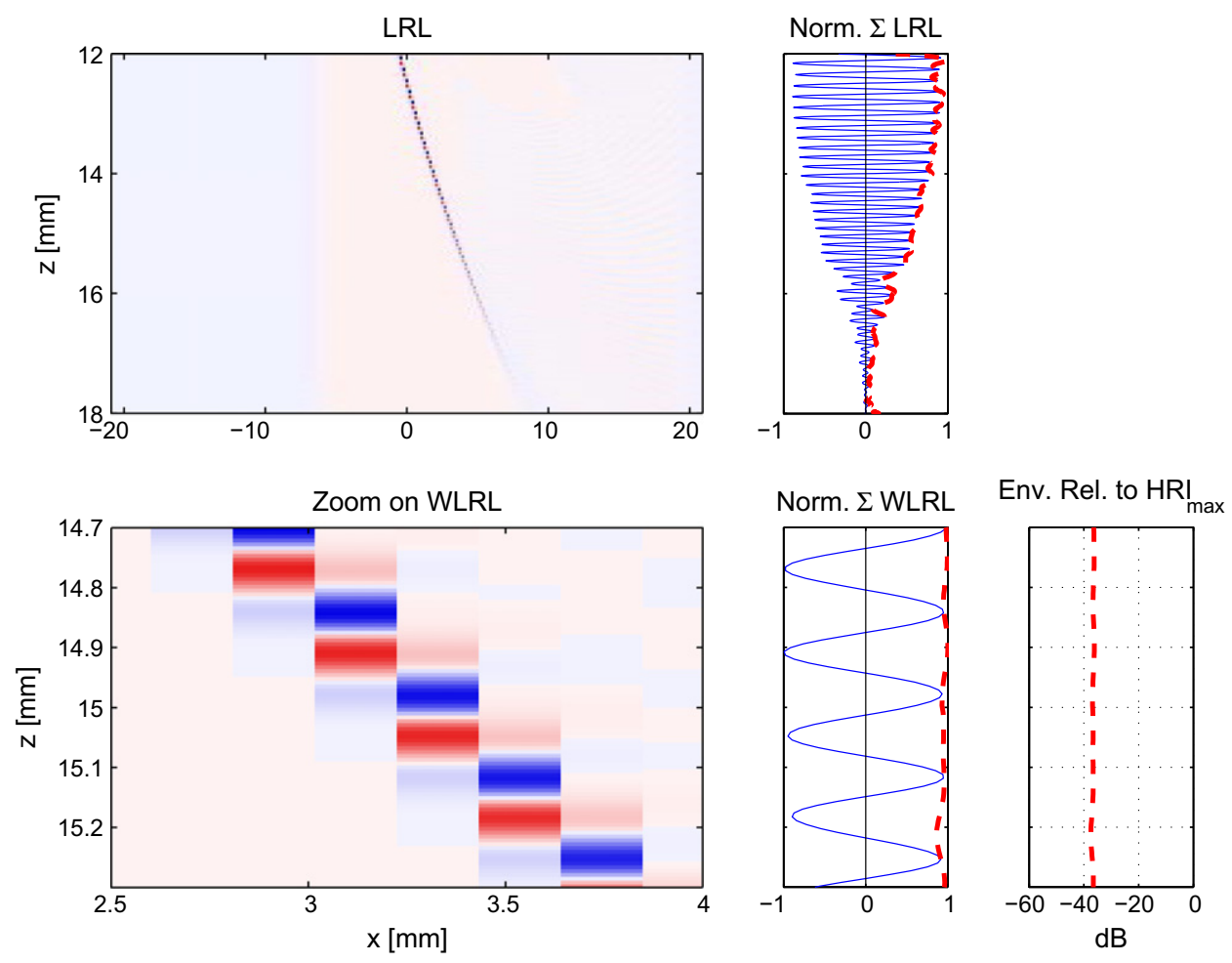

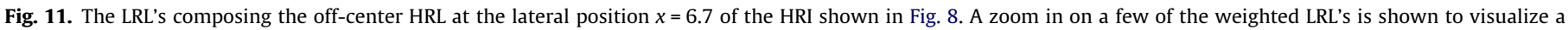
phase-shift of almost an entire pulse-echo wave length, yielding undesired constructive summation, and an envelope level above - $40 \mathrm{~dB}$.
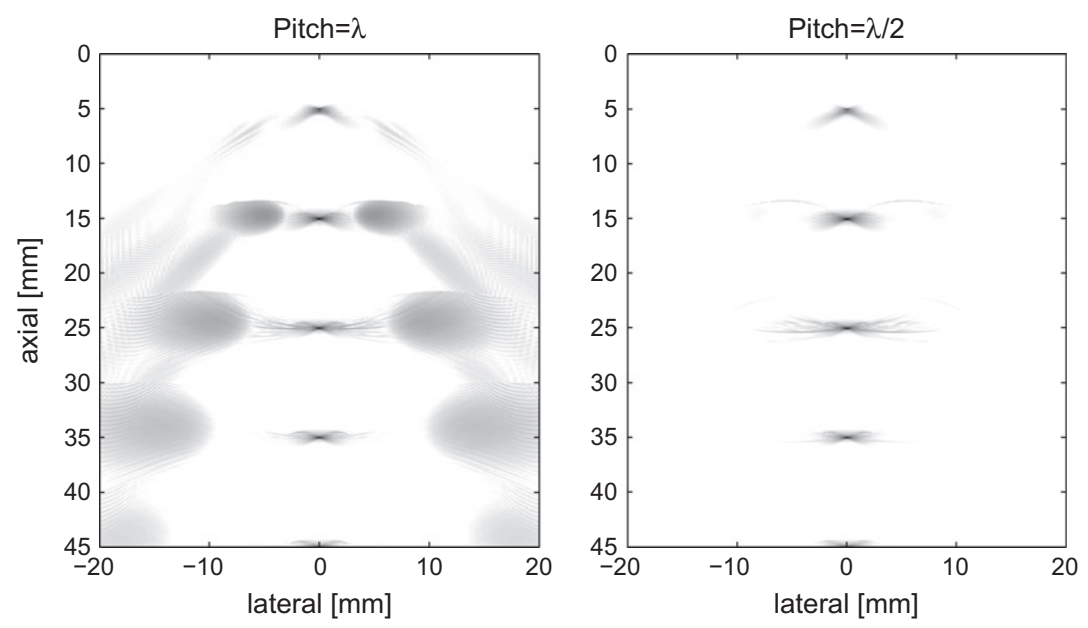

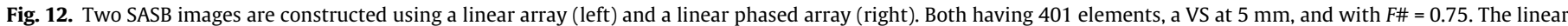
array is twice the width of the phased array, but the image has been cropped to the same width as the phased array image. Dynamic range is $70 \mathrm{~dB}$.

The PSF at the output of the 1 st stage beamformer has a large extent because of the fixed receive focusing. The curvature is determined by the distance between the VS's and the point of the PSF. Consider the point at position $\vec{r}_{p}=(x, z)=\left(0, z_{p}\right)$. The curvature, $z_{c}(x)$ is

$z_{c}(n)=z_{v} \pm \sqrt{\left(z_{p}-z_{v}\right)^{2}+(|n| \Delta)^{2}}$.

The variable $x$ has been substituted by $n \Delta$, where $-N / 2 \leqslant n \leqslant N / 2$ is the VS number counting from the VS at $x=0$. The \pm refer to whether the image point is above or below the VS, just as in (3), and (8). The contour plot of the PSF is shown in Fig. 5 with a VS at $5 \mathrm{~mm}$ and with $F \#=0.5$, and $F \#=2$. The shape of the PSF is the same in both cases, but the width of the PSF differs due to the different opening angles. $z_{c}(n)$ from (10) has been plotted on top of the contour plot to illustrate the coherence. A PT is placed at $z=65 \mathrm{~mm}$ and $z_{c}$ has been plotted for two points at $z_{p}=64 \mathrm{~mm}$ and $z_{p}=66 \mathrm{~mm}$, respectively. The curvatures of these plots are similar to the PSF.

The envelope images after 2nd stage processing of these data sets are shown in Fig. 6 with a $60 \mathrm{~dB}$ dynamic range. The images are constructed with $N=401$ which equals an image width of approximately $83 \mathrm{~mm}$, but the displayed images have been cropped to a width of $40 \mathrm{~mm}$. The resolution seems range independent if the dominant side-lobes are discounted. The lateral 
resolution decreases as the $F \#$ increases as expected because of the decrease in the width of the synthesized aperture.

The quantified resolution of the HRI in Fig. 6 is shown in Fig. 7. The resolution is practically constant at $-6 \mathrm{~dB}$ and $-40 \mathrm{~dB}$ through range for all values of $F \#$, with a few exceptions. The exceptions are for those PT near the VS when $F \#=0.5$. Here the side-lobe level is very dominant. Side-lobes are also noticeable for $F \#=1$ when looking at the HRI in Fig. 6. They are below the $-40 \mathrm{~dB}$ level though and do not show in Fig. 7.

\subsubsection{Investigating grating-lobes}

The off-axis energy-lobes in Fig. 6 for $F \#=0.5$ are dominant Taking a closer look at the phase of those LRL's which are summed in the 2nd stage beamformer can disclose the origin of this artifact. The part of the image containing the PT at $15 \mathrm{~mm}$ is shown in Fig. 8. Three different HRL's are investigated. They are marked with dotted lines in Fig. 8. SASB relies on phase coherent addition of the LRL's as expressed in (4). The LRL's composing the center HRL are plotted in Fig. 9. It is apparent that the LRL's are completely phase aligned and add up constructively as expected. The figure shows the LRL's before and after the weighting function. It also shows the HRL, hence the summation of the LRL's and the corresponding envelope. Finally the envelope of the weighted HRL is shown relative to the maximum of the HRI in Fig. 8.

The LRL's composing the HRL at $x=3.1 \mathrm{~mm}$ are plotted in Fig. 10. The LRL's are shown before the weighting and a zoom in on a few of the LRL's after the weighting function is shown. The phase shift between consecutive LRL's is close to half a pulse-echo wave length and this destructive summation yields an envelope level near $-60 \mathrm{~dB}$.

The LRL's composing the HRL at $x=6.7 \mathrm{~mm}$ are plotted in Fig. 11. The phase shift between consecutive LRL's has increased to almost an entire pulse-echo wave length and the LRL's add up constructively to an envelope level above $-40 \mathrm{~dB}$. In this simulated setup the pulse-echo signal consists of only a few periods, hence only a few non-zero contributions are included in the summation of the LRL's. If the pulse-echo signal contains several periods

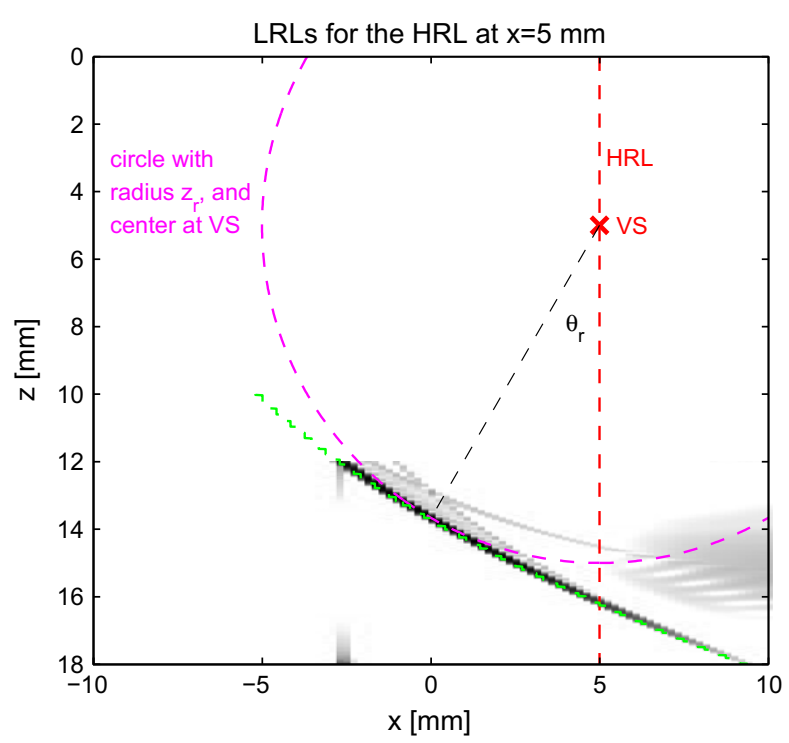

Fig. 13. The log-compressed envelope of the LRL's constituting the HRL at $x_{h}=4 \mathrm{~mm}$ is shown in the range $12-18 \mathrm{~mm}$ with a dynamic range of $50 \mathrm{~dB}$. The VS is at the depth $z_{v}=5 \mathrm{~mm}$ and $F \#=0.5$. A PT is positioned at the point $\vec{r}_{p}=(x, y)=\left(0, z_{p}\right)$ with depth, $z_{p}=15 \mathrm{~mm}$. The appearance of the LRL's for the offcenter HRL can be approximated by a rotation of the LRL's of the center HRL. The center of rotation is the VS of the off-center $\mathrm{HRL}$, and the rotation radius is $z_{r}=z_{p}-z_{v}$. The angle of rotation $\theta_{r}=\arcsin \left(x_{h} / z_{r}\right)$ is also indicated. several non-zero LRL's will be present in the sum, yielding an even higher envelope level.

The artifacts shown are grating lobes, since they arise due to lateral spatial under-sampling. Consider having a setup with a phased array transducer with a $\lambda / 2$ pitch and, thus, twice the lateral density of VS's. The illustration of LRL's prior to summation equivalent to the one shown in Fig. 11 will contain LRL's with a phase shift between consecutive LRL's of only half a wave length. Every second LRL will be equal to the ones shown in Fig. 11 but every other LRL will have a phase which is in between the two. The result is destructive summation instead of unintentional constructive summation, and the massive grating lobes are avoided. This is exemplified in Fig. 12. Two HRI's are constructed using a linear array with $\lambda$-pitch and a linear phased array with $\lambda / 2$-pitch respectively. The
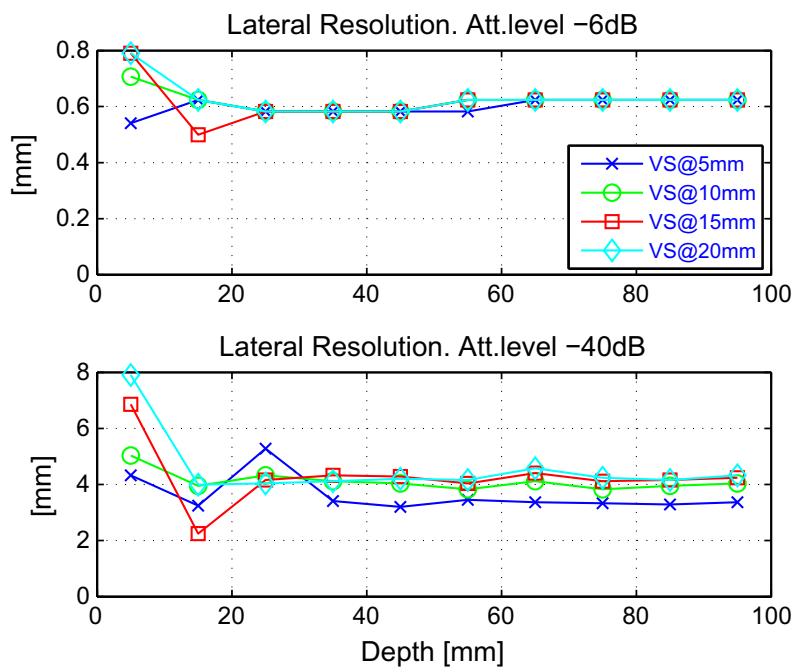

Fig. 14. Lateral resolution with $F \#=1.5$ as a function of depth at $-6 \mathrm{~dB}$ (top) and $-40 \mathrm{~dB}$ (bottom).
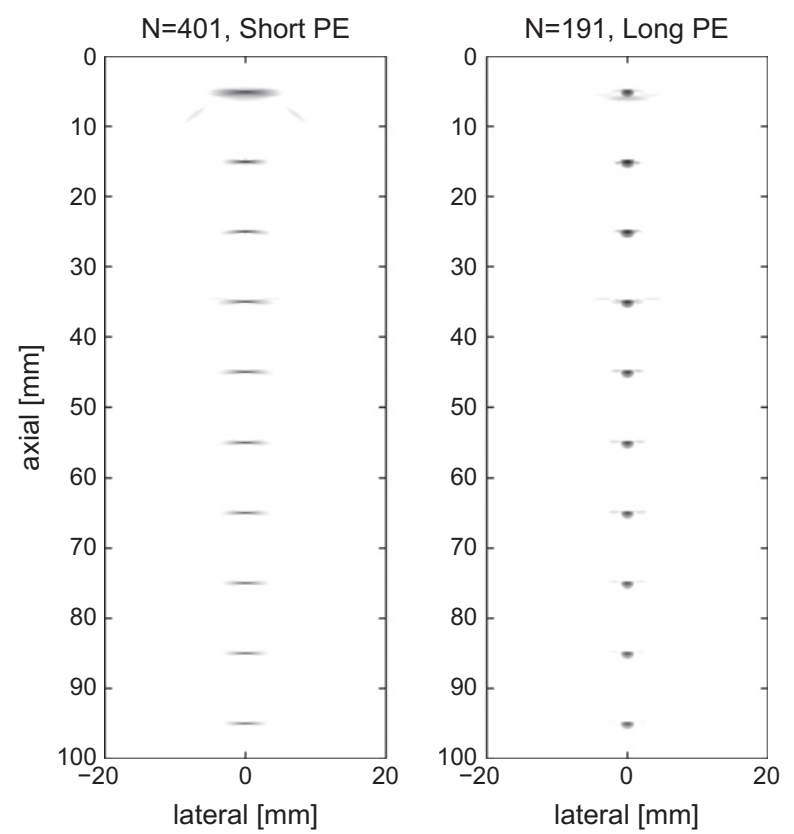

Fig. 15. Envelope images with the VS at $20 \mathrm{~mm}$ and with $F \#=2$. Two different simulation models are used. $N=401$, and a short pulse-echo (PE) response (left). $N=191$, and a long pulse-echo response (right). The image using $N=401$ has been cropped to the same width as the image using $N=191$. Dynamic range is $60 \mathrm{~dB}$. 

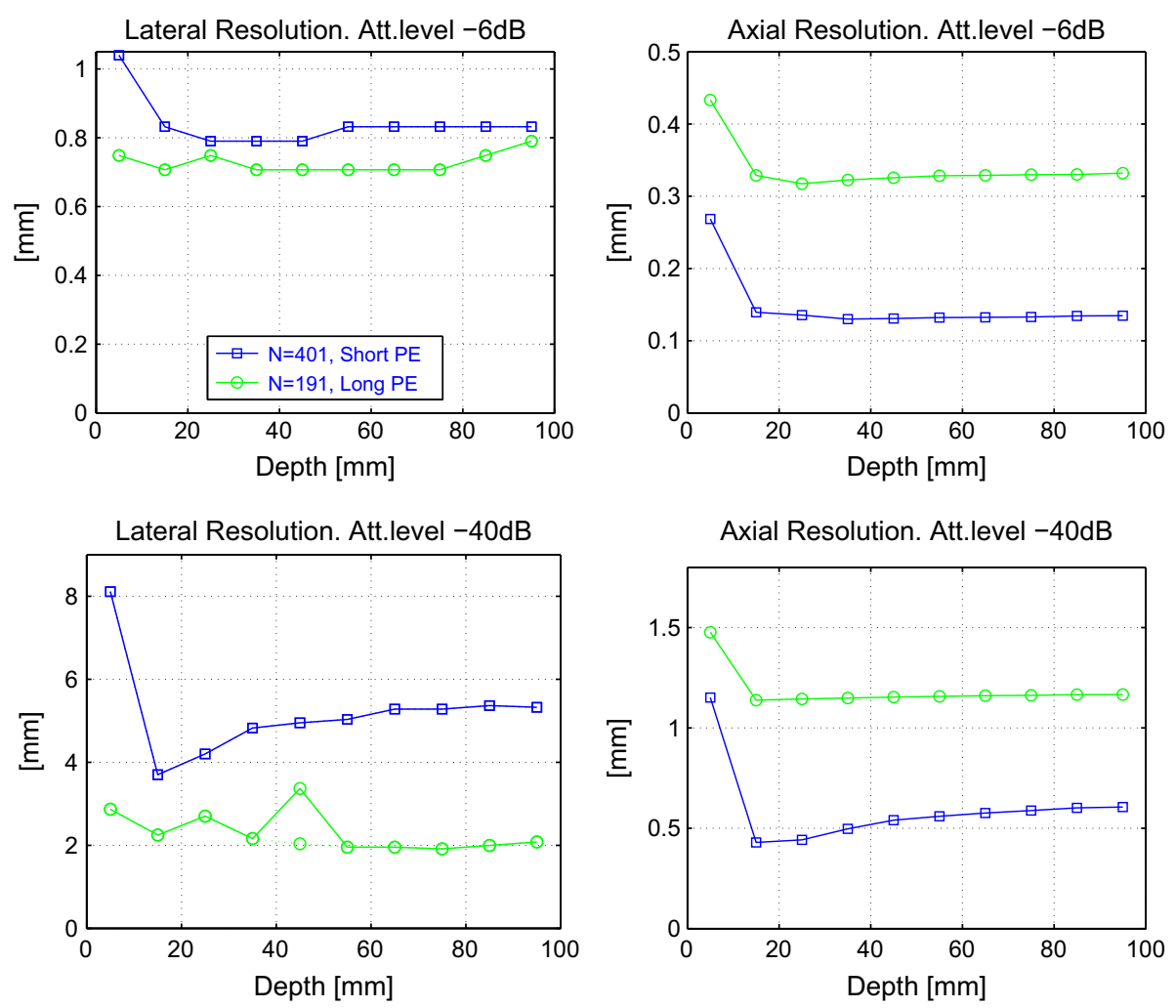

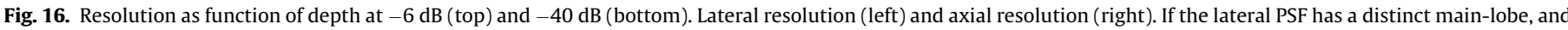
side-lobe distribution, the main-lobe resolution is shown as a dotted line. The resolution is extracted from the HRI's in Fig. 15.

difference between the two images is striking, since the grating lobes are avoided by increasing the lateral spatial sampling in the 1st stage processing.

The VS's can be conceived as elements in a virtual array. In SASB the wave fields from several emissions are sampled by the virtual array and coherently added. For a given frequency grating lobes arise at a combination of a sparse spatial sampling by the virtual array and wave fields with incident angles beyond a certain limit. Both of these parameters can be controlled in SASB to prevent grating lobes. The array spacing and the VS spacing do not have to be the same. With a dense lateral sampling the VS can have $\lambda / 2$ spacing even though the array has $\lambda$ spacing. For a given spacing between the VS's the range of incident angles must be restricted. This is possible by putting a limit to the opening angle of the VS.

The restriction on the opening angle to avoid grating lobes can be demonstrated from an analysis of the LRL's constituting the offcenter HRL's. In Fig. 9 the LRL's constituting the center HRL are phase-coherently aligned. They are aligned at the depth, $z_{p}=15 \mathrm{~mm}$, where a PT at $\vec{r}_{p}=(x, y)=\left(0, z_{p}\right)$ is placed. In Fig. 11 the LRL's constituting an off-center HRL at the lateral position $x_{h}$ are no longer aligned phase-coherently. As a simple approximation these LRL's can be found by a rotation of the aligned LRL's. The line of phase equality is perpendicular to the line with origin in the VS of the off-center HRL and going to $\vec{r}_{p}$ and with length $z_{r}=z_{p}-z_{v}$, where $z_{v}$ is the depth of the VS. The angle of this line is

$\theta_{r}=\arcsin \left(\frac{x_{h}}{z_{r}}\right)$

This geometry is illustrated in Fig. 13 superimposed on the logcompressed envelope of a set of LRL's from the HRL at $x_{h}=5 \mathrm{~mm}$. The exact positions of constant phase are also indicated in Fig. 13 as a dashed line. It comes from the intersections of the indices representing the PT and the indices representing a number of FP's all placed in the same off-center HRL.

By applying the approximation about the rotated LRL's it is possible to estimate the phase shift between consecutive LRL's with a simple expression. The axial shift between consecutive LRL's is

$x=\tan \theta_{r} \Delta$.

Substituting $\theta_{r}$ with half the opening angle, $\alpha^{\prime}=\alpha / 2$ the axial shift can be expressed as

$x=\tan \left(\alpha^{\prime}\right) \Delta=\frac{\Delta}{2 F \#}$

If the spatial shift does not exceed half the wavelength of the pulseecho signal, $\lambda_{p e}=\lambda / 2=c / f_{0} / 2$ grating lobes are avoided. That is
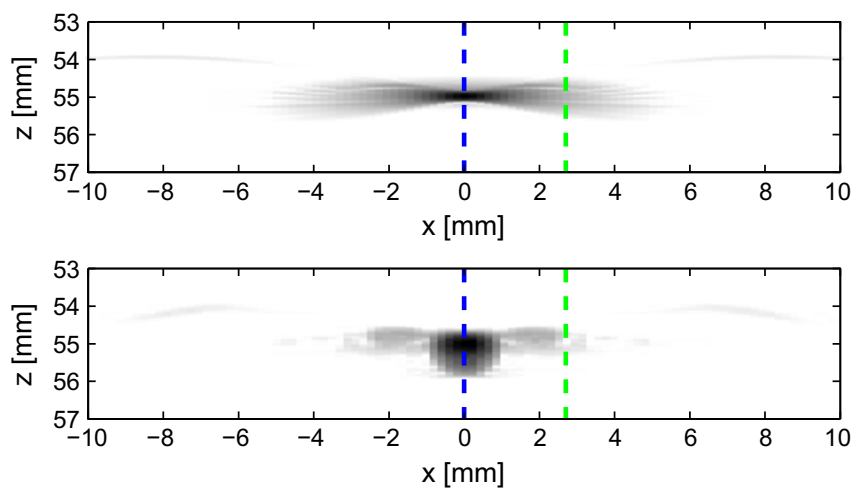

Fig. 17. Envelope images with the VS at $10 \mathrm{~mm}$, and with $F \#=2$ from Fig. 15 . Short PE response (top), and long PE response (bottom). Shown in the range from $53 \mathrm{~mm}$ to $57 \mathrm{~mm}$, and superimposed with vertical dashed lines indicating those HRL's for which the LRL's are shown. The LRL's are shown in Figs. 18 and 19. 


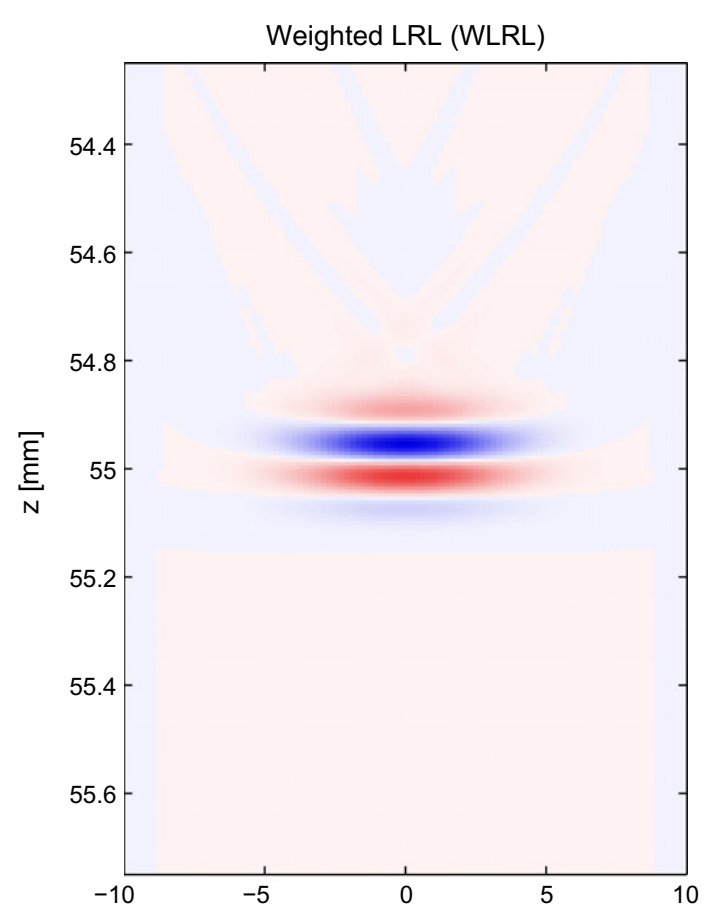

Norm. $\Sigma$ WLRL

Env. Rel. to HRI ${ }_{\max }$
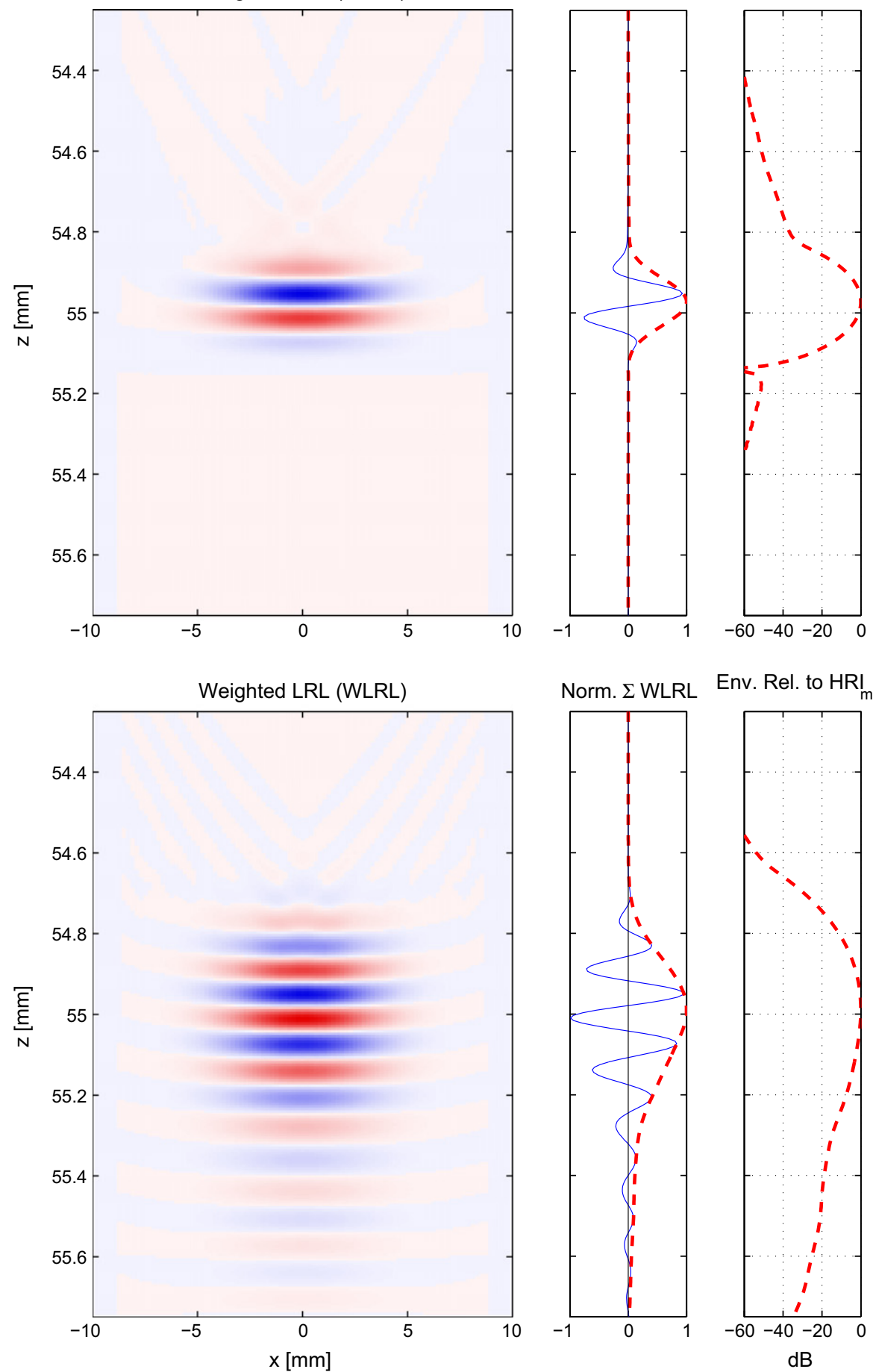

Norm. $\Sigma$ WLRL

Env. Rel. to $\mathrm{HRI}_{\text {max }}$
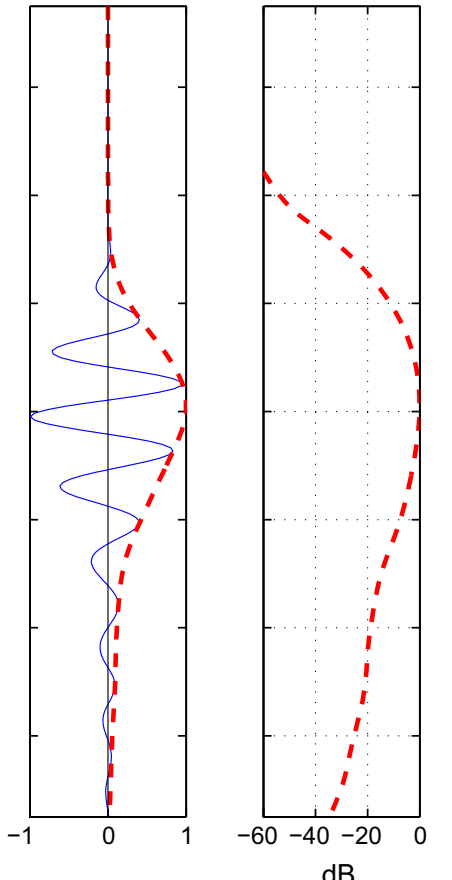

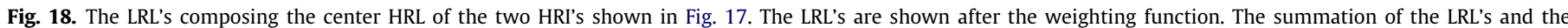
corresponding envelope are also shown.

$x<\lambda_{\text {pe }} / 2$ or $x<\lambda / 4$ This condition can be used to put restrictions on the $F \#$ of the VS

$F \# \geqslant \frac{\Delta}{\lambda / 2}$ for $x \leqslant \lambda / 4$

The opening angle of the VS limits the range of angles of the wave field. If a transducer with $\Delta=\lambda$ is used in SASB grating lobes are avoided if $F \# \geqslant 2$.
The spatial pulse-echo wavelength is extracted from the center LRL and is $\lambda_{\text {pe }}=0.15 \mathrm{~mm}$. With $\Delta=0.208 \mathrm{~mm}$ the VS must be designed with $F \# \geqslant 1.39$ with the restriction of (14). This is also reflected in Fig. 6, where the grating lobes evidently are attenuated for $F \#=1.5$ and $F \#=2$ compared to the setup where $F \#=0.5$, and $F \#=1$. In Fig. 12 (right) a phased array with pitch $\Delta=0.104$ is applied, which requires $F \# \geqslant 0.70$. The applied $F \#$ is 0.75 and the grating lobes are avoided. Notice that the requirement for the F\# is not tied to the array spacing but to the spacing of the VS's. The 


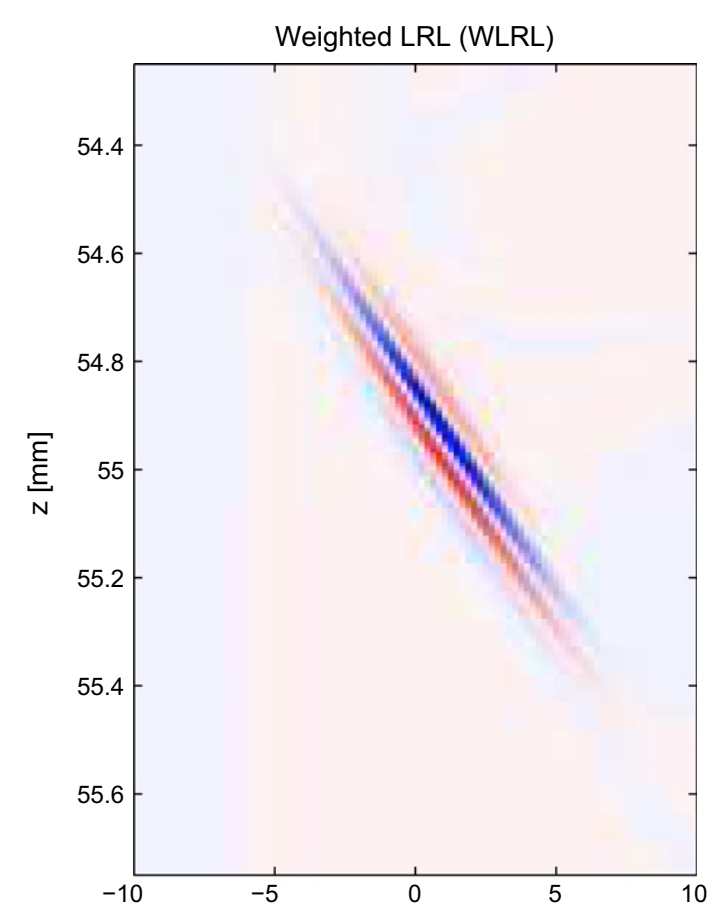

Norm. $\Sigma$ WLRL

Env. Rel. to $\mathrm{HRI}_{\max }$
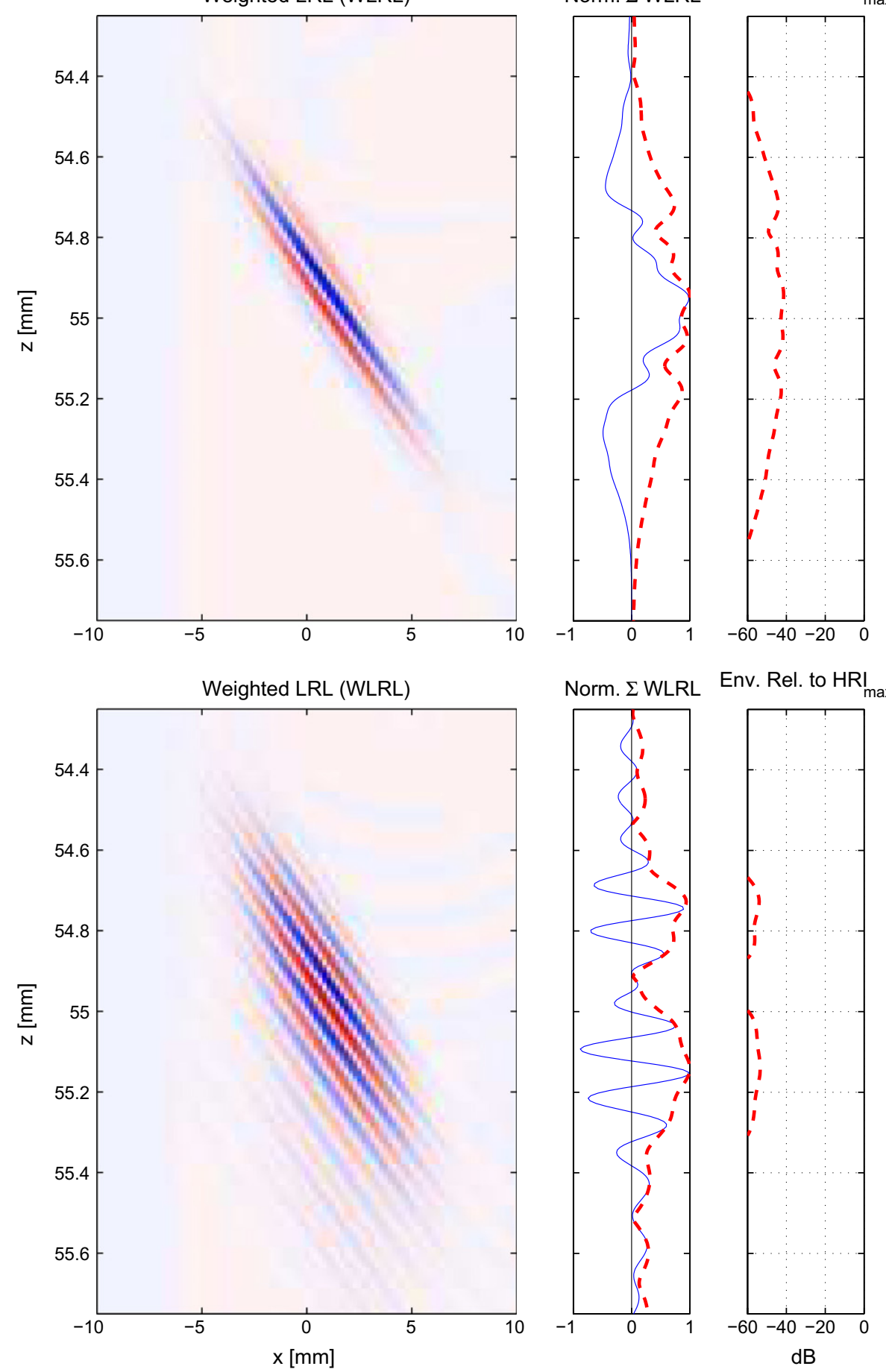

Norm. $\Sigma$ WLRL

Env. Rel. to $\mathrm{HRI}_{\max }$
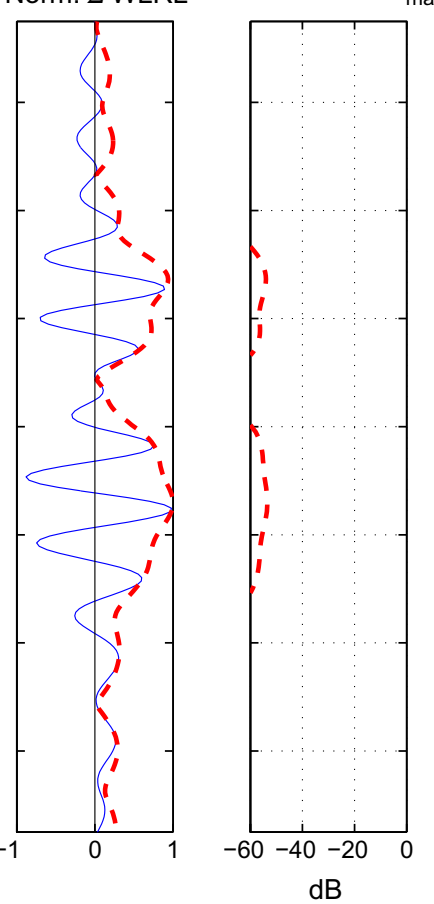

Fig. 19. The LRL's composing the off-center HRL of the two HRI's shown in Fig. 17. The LRL's are shown after the weighting function. The summation of the LRL's and the corresponding envelope are also shown.

setup is thus equivalent to a setup where $\Delta=0.208$ and the density of the VS's is doubled.

$K(z)$ from (5) determines the number of VS's applied and the size of the synthesized aperture. It is a function of the position of the VS and the opening angle and, thus, the F\#. It was shown previously that the grating lobes were appropriately attenuated for the linear array with $F \#=1.5$ when the VS was at $5 \mathrm{~mm}$. The lateral resolution with $F \#=1.5$ and different positions of the VS is shown
Table 2

The number of array elements used during transmission as a function of VS position and $F \#$.

\begin{tabular}{lll}
\hline VS depth & F\# $=1.5$ & $F \#=2$ \\
\hline $5 \mathrm{~mm}$ & 17 & 13 \\
$10 \mathrm{~mm}$ & 33 & 25 \\
$15 \mathrm{~mm}$ & 49 & 37 \\
$20 \mathrm{~mm}$ & 65 & 49 \\
\hline
\end{tabular}


in Fig. 14. The resolution functions are very similar but not the same in the four different setups because of the different number of applied VS's.

\subsubsection{Influence of transducer impulse response}

The performance results presented in the previous sections were based on a transducer with $N=401$, a short excitation signal, and a simple impulse response. The performance using a more realistic transducer simulation model is presented in this section and a comparison between the two is made. Envelope images with the VS at $20 \mathrm{~mm}, F \#=2$, and with the two different simulation models are shown in Fig. 15. The new simulation model has $N=191$. The excitation signal has two periods, and the impulse response is the measured response of a commercial transducer yielding a longer pulse-echo (PE) response. The difference between the two images is significant. The axial resolution has decreased as a consequence of the extended PE response. The lateral resolution has improved in the entire range also beyond depths of $80 \mathrm{~mm}$, where $K(z)$ ceases to increase linearly. The axial and lateral resolution are extracted, and shown in Fig. 16. The improvement in lateral resolution at $-40 \mathrm{~dB}$ is at least a factor of 2 .

To illustrate the cause of the difference in performance, the LRL's composing the HRI's are investigated. The investigation
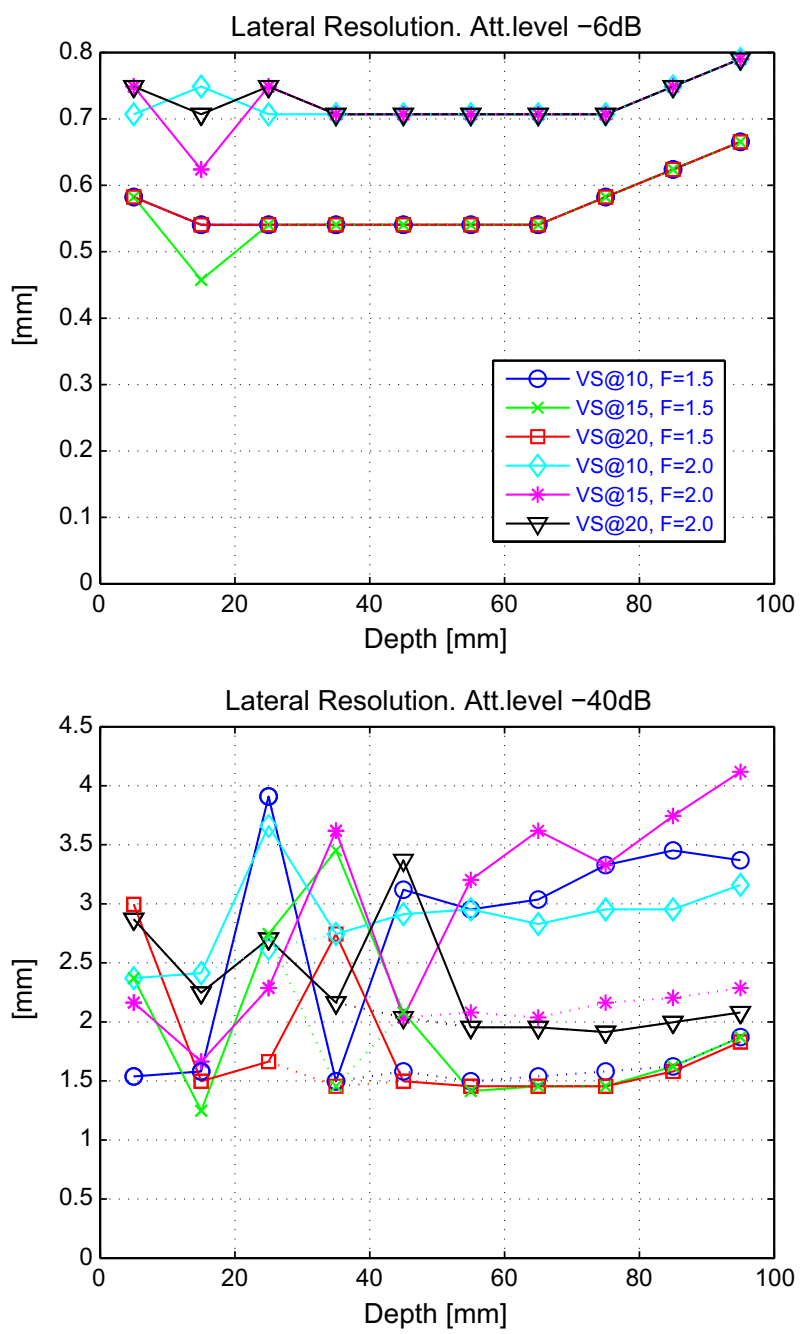

Fig. 20. Lateral resolution of SASB as function of depth at $-6 \mathrm{~dB}$ (top) and $-40 \mathrm{~dB}$ (bottom). If the lateral PSF has a distinct main-lobe, and side-lobe distribution, the main-lobe resolution is shown as a dotted line. In all configurations the results are based on the realistic transducer simulation model introduced in Section 3.2.2. concerns only the range from $53 \mathrm{~mm}$ to $57 \mathrm{~mm}$ which include a single PT at a depth of $55 \mathrm{~mm}$. The HRI's in this range are shown in Fig. 17, superimposed with vertical dashed lines indicating those HRL's for which the LRL's are shown.

The LRL's for the center HRL are shown in Fig. 18. The LRL's for the off-center HRL are shown in Fig. 19. In these figures the LRL's after LRL-weighting are shown. They also show the summation of the LRL's and the corresponding envelope. Finally the envelope of the weighted HRL is shown relative to the maximum of the HRI's in Fig. 17. The LRL's sum completely constructive for the center HRL in both setups.

For the off-center HRL's the LRL's sum destructively toward zero level. With a short pulse-echo response the off-center HRL is composed of the sum of a only a few positive and negative half-periods. The amplitudes of these half-periods varies with range and lateral position. The amplitude of the HRL, thus, varies with range. The variation over range is considerable due to the sparse number of cycles in the sum.

With a longer pulse-echo response the off-center HRL is composed of the sum of several positive and negative half-periods. The amplitudes of these half-periods decay smoothly over range and lateral position and on average sum destructively to a zero level. The HRL is of oscillating nature with the same center frequency as the pulse-echo response, which is not the case for the setup with the short PE response.

\subsubsection{Comparison to dynamic receive focusing}

The performance of SASB is among others a function of VS position and $F \#$. These parameters also determine the number of elements used during transmission. This has an influences on the emitted energy and the signal to noise ratio. It was shown in Section 3.2.1 that the grating lobes were appropriately attenuated for $F \#=1.5$ and $F \#=2$. The number of elements used as a function of VS position and $F \#$ is shown in Table 2.

The choice of configuration for comparison with DRF is based on the results presented in Fig. 20. Here the lateral resolution for a number of configurations is shown. In all configurations the results are based on the realistic transducer simulation model introduced
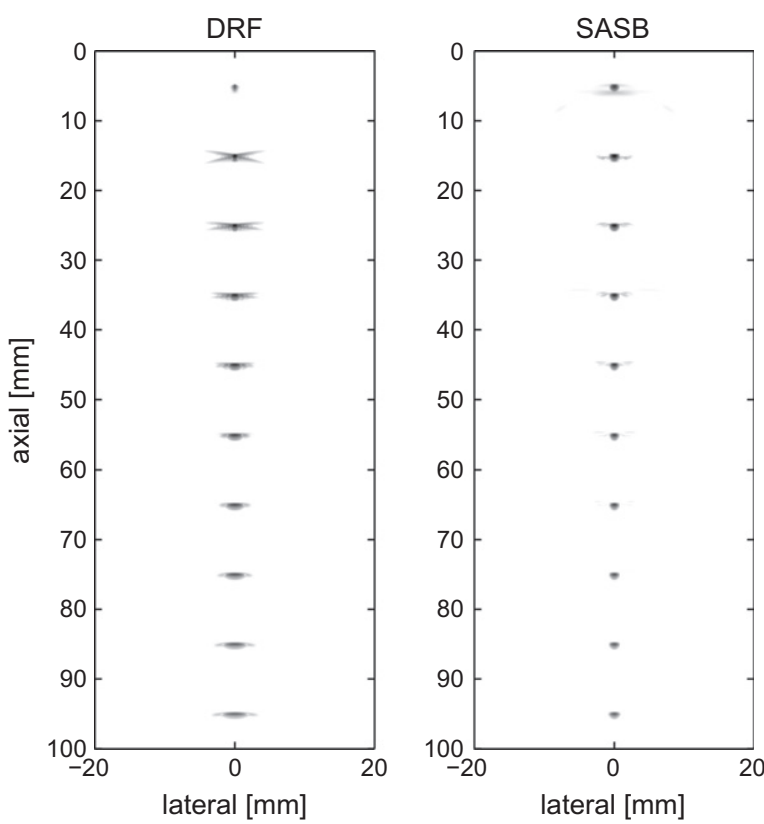

Fig. 21. Envelope images using DRF (left) and SASB (right). For DRF the transmit focal point is at $70 \mathrm{~mm}$. For SASB the VS is at $20 \mathrm{~mm}$ and $F \#=1.5$. Dynamic Range is $60 \mathrm{~dB}$. 

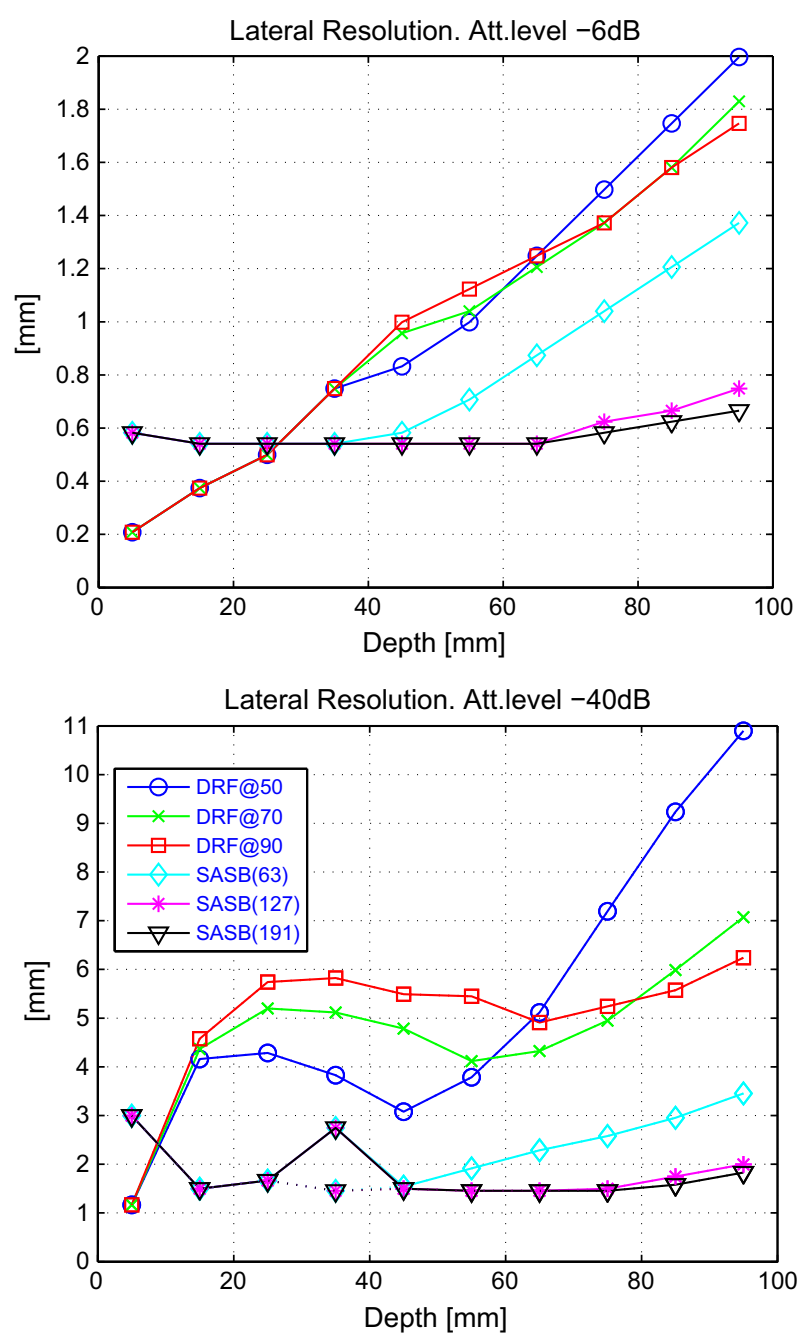

Fig. 22. Lateral resolution of DRF and SASB as function of depth at $-6 \mathrm{~dB}$ (top) and $-40 \mathrm{~dB}$ (bottom). If the lateral PSF has a distinct main-lobe, and side-lobe distribution, the main-lobe resolution is shown as a dotted line. In all configurations the results are based on the realistic transducer simulation model introduced in Section 3.2.2. For DRF the transmit focal point is at $50 \mathrm{~mm}, 70 \mathrm{~mm}$, and $90 \mathrm{~mm}$. For SASB the VS is at $20 \mathrm{~mm}$ and $F \#=1.5$. SASB results are presented using different number of available 2 nd stage beamformer channels. $N_{2 n d}=63, N_{2 n d}=127$, and $N_{2 n d}=191$.

in Section 3.2.2. The performance with the VS at $20 \mathrm{~mm}$ and $F \#=1.5$ is superior to the other configurations in almost the entire range. It is thus a rational choice for a good performance and a comparison with DRF.

Fig. 21 shows images with DRF and SASB side by side, and Fig. 22 shows the quantified lateral resolution for different configurations. The quantified axial resolution does not differ between the different configurations and is not shown. Different positions of the transmit focal point in DRF has been applied for a fair comparison. The VS is at $20 \mathrm{~mm}$ and $F \#=1.5$ for all cases of SASB. In Fig. 21, and in the previous sections the number of channels in the 2nd stage beamformer is unlimited, so that $N_{2 n d}=N$. In Fig. 22 additional SASB results are presented where the number of channels has been limited to $N_{2 n d}=127$, and $N_{2 n d}=63$.

There is an substantial improvement in resolution using SASB compared to DRF. It accounts for both the FWHM and the resolution at $-40 \mathrm{~dB}$. The improvement in FWHM is at least a factor of 2 and the improvement at $-40 \mathrm{~dB}$ is at least a factor of 3 . The improvement of SASB over DRF is a reality except for a few exceptions. At depths until $20 \mathrm{~mm}$ the FWHM is superior with DRF. With SASB the resolution is almost constant throughout the range. For
DRF the FWHM increases almost linearly with range and the resolution at $-40 \mathrm{~dB}$ is fluctuating with range.

By putting restrictions on the number of 2nd stage beamformer channels the system complexity is reduced. It will have a negative consequence on resolution, since the synthesized aperture decreases. When the number of channels is restricted to $N_{2 n d}=63$, and $N_{2 n d}=127$ then the depths at which $K(z)$ ceases to increase linearly is at $40 \mathrm{~mm}$ and $61 \mathrm{~mm}$ respectively. This is also apparent when observing the resolution in Fig. 22 when these restrictions are applied. Both the FWHM and the resolution at $-40 \mathrm{~dB}$ cease to be constant at these depths. Even when the number of channels is restricted to $N_{2 n d}=63$ the performance of SASB is superior to DRF.

\section{Measurement results}

A commercial scanner and a linear array transducer with parameters similar to the ones in Table 1 have been used to acquire data. A tissue phantom with wire targets and $0.5 \mathrm{~dB} / \mathrm{MHz} / \mathrm{cm}$ attenuation is used as imaging object. RF-data using DRF and 1st stage SASB is acquired with a $5 \mathrm{MHz}$ center frequency and stored. 2nd stage SASB processing, envelope detection, and logarithmic compression is done off-line for both DRF and SASB. An envelope image of the acquired 1st stage SASB data is shown in Fig. 23. It resembles the shape of the contours shown in Fig. 5 as expected. Notice the arcs are turned upside down before and after the focal point at $20 \mathrm{~mm}$. A side by side comparison between the DRF image and the SASB image is also shown in Fig. 23. With DRF the transmit

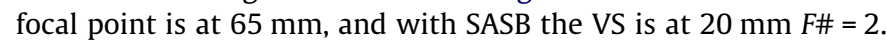

The images based on measured data confirms the results from the simulations. At the center of the image the resolution of SASB is superior to DRF and is practically range independent. A zoom in on the PT at $69 \mathrm{~mm}$ for the DRF and SASB images from Fig. 23 is shown in Fig. 24. Here the difference in resolution is prominent. The resolution in the near field is slightly better for DRF as stated in Section 3.2.3 and in Fig. 22.

The speckle pattern is present at a greater range in the SASB image compared to the DRF image indicating greater penetration. At greater depths the emitted wave field in SASB is wide compared to DRF. The intensity is lower and so is the SNR of the 1st stage SASBdata. The SNR increases because of the summation of a number of LRL's. Provided that the image object does not move in between acquisitions and assuming uncorrelated electronic noise at the receivers the potential SNR improvement at the center HRL compared to 1 st stage RF-data is $\Delta S N R(z)=10 \log _{10}(K(z))$ with a rectangular apodization in the 2nd stage. With an apodization function $\mathcal{W}$ the SNR improvement becomes

$\Delta \operatorname{SNR}(z)=10 \log _{10}\left(\sum_{k=1}^{K(z)} \mathcal{W}\left(x_{k}, z\right)\right)$

The signal to noise ratio as a function of depth for a set of acquired DRF-data and a set of 1st stage SASB-data has been estimated, and is shown in Fig. 25. For DRF the transmit focus is at $45 \mathrm{~mm}$ and for SASB the VS is at $20 \mathrm{~mm}$ with $F \#=2.20$ data sets have been acquired for both DRF and SASB using a tissue phantom with an attenuation of $0.5 \mathrm{~dB} /[\mathrm{MHz} \mathrm{cm}]$. The signal part is estimated by averaging the measured signals. A set of noise signals is estimated by subtracting the estimated signal part from the measured signals. The SNR is

$$
\operatorname{SNR}(z)=\frac{\mathcal{E}\{Y(z)\}^{2}}{\mathcal{E}\{[Y(z)-\mathcal{E}\{Y(z)\}]\}^{2}} \simeq \frac{\left(\frac{1}{M} \sum_{m=1}^{M} y_{m}(z)\right)^{2}}{\frac{1}{M} \sum_{m=1}^{M}\left[y_{m}(z)-\frac{1}{M} \sum_{m=1}^{M} y_{m}(z)\right]^{2}} .
$$



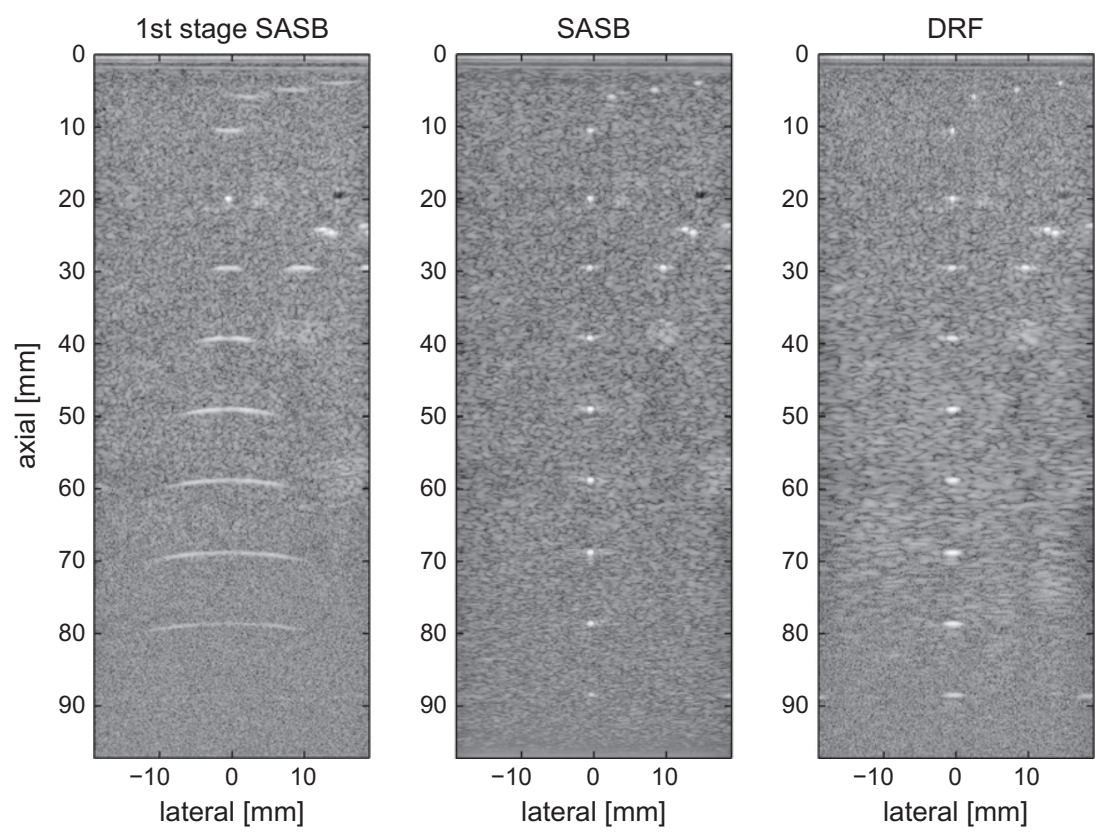

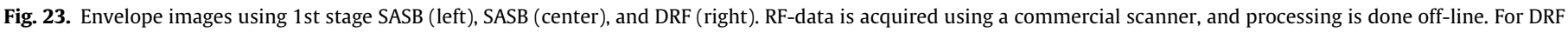
the transmit focal point is at $65 \mathrm{~mm}$. For SASB the VS is at $20 \mathrm{~mm}$ and $F \#=2$. Dynamic Range is $60 \mathrm{~dB}$.
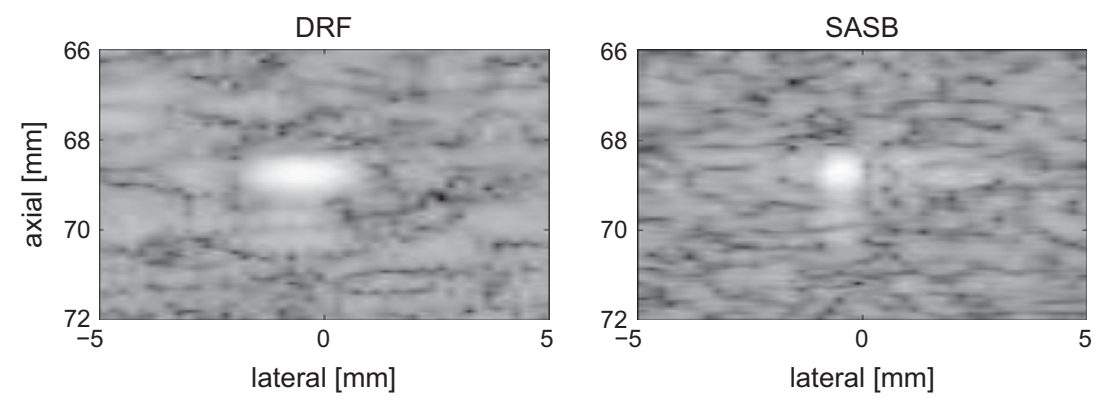

Fig. 24. A zoom in on the DRF and SASB images from Fig. 23.

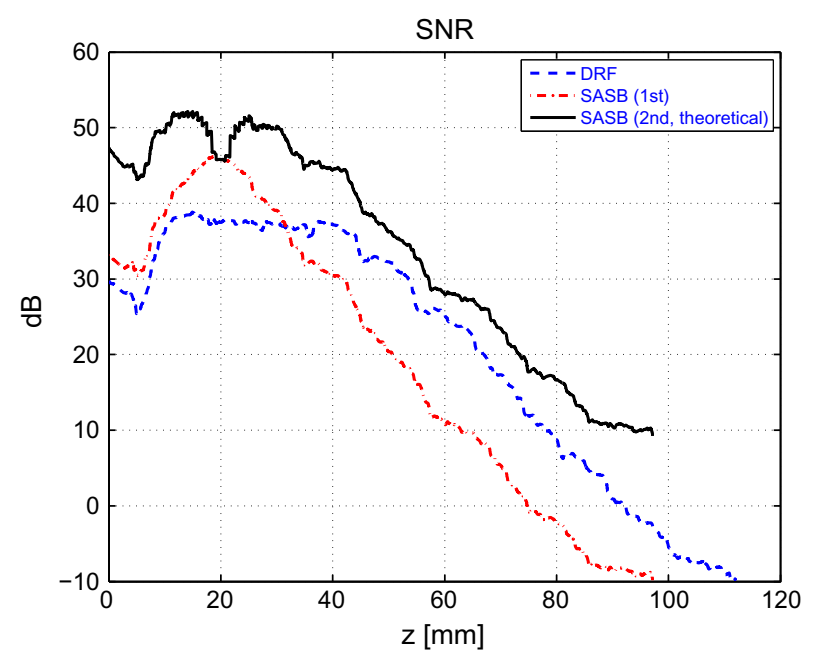

Fig. 25. SNR as a function of depth. Data sets are acquired with a commercial scanner and a tissue phantom for both DRF and 1 stage SASB. The estimated SNR of 2nd stage SASB is also shown.
$\mathcal{E}$ is the expectation operator. $Y(z)$ denotes the $M$ number of measured data sets, and $y_{m}(z)$ is the $m$ 'th data set. The theoretical potential SNR improvement, $\triangle S N R(z)$ from (15) is also shown in Fig. 25 with a Hamming window as SA weighting. For $z=80 \mathrm{~mm}$ the value of $\Delta S N R(z)$ can be calculated using (6) and (5) and is $19 \mathrm{~dB}$. The potential SNR of the SASB is superior to DRF at the entire range and an improvement of $8 \mathrm{~dB}$ is realized at a depth of $80 \mathrm{~mm}$.

\section{Conclusion}

The main motivation for SASB is to apply synthetic aperture techniques with a restricted system complexity. The objective is to improve lateral resolution and obtain a more range independent resolution compared to conventional ultrasound imaging.

The SASB method has been investigated using simulations in Field II and by off-line processing of data acquired with a commercial scanner. The parameters which are the most decisive for the performance are the depth of the VS (the focal depth), and the $F \#$ of the VS. The lateral resolution increases with a decreasing $F \#$, but grating lobes arise at a combination of a sparse spatial sampling by the virtual array and wave fields with incident angles 
beyond a certain limit. It is possible to restrict the range of incident angles by putting a limit to the opening angle of the VS. For a transducer with a pitch of $\Delta=\lambda$ grating lobes are avoided if $F \# \geqslant 2$. For a phased array transducer with $\Delta=\lambda / 2$ grating lobes are avoided if $F \# \geqslant 1$.

Simulations have been done with two different pulse-echo responses having different bandwidths. The performance difference between the two simulation models is significant. The lateral resolution is significantly better for the simulation model with the longer pulse-echo response and the improvement is at least a factor of 2 at $-40 \mathrm{~dB}$.

The performance of SASB with the VS at $20 \mathrm{~mm}$ and $F \#=1.5$ is compared with conventional dynamic receive focusing (DRF). The axial resolution is the same for the two methods. There is an substantial improvement in lateral resolution using SASB compared to DRF. It accounts for both the FWHM and the resolution at $-40 \mathrm{~dB}$. The improvement in FWHM is at least a factor of 2 and the improvement at $-40 \mathrm{~dB}$ is at least a factor of 3 . The improvement of SASB over DRF is a reality except for a few exceptions. At depths until $20 \mathrm{~mm}$ the FWHM is superior with DRF. With SASB the resolution is almost constant throughout the range. For DRF the FWHM increases almost linearly with range and the resolution at $-40 \mathrm{~dB}$ is fluctuating with range. By putting restrictions on the number of 2 nd stage beamformer channels the system complexity is reduced. It will have a negative consequence on resolution since the synthesized aperture decreases, but even when the number of channels is restricted to $N_{2 n d}=63$ the performance of SASB is still superior to DRF.

SASB has been applied to data acquired with a commercial scanner and a tissue phantom with wire targets. The images confirms the results from the simulations. At the center of the image the resolution of SASB is superior to DRF and is practically range independent. The resolution in the near field is slightly better for DRF. A feasible solution could be to construct the final image by applying DRF above the VS and SASB beyond the VS.

The speckle pattern is present at a greater range in the SASB image compared to the DRF image indicating greater penetration. The signal to noise ratio as a function of depth for a set of acquired DRFdata and a set of 1 st stage SASB-data has been estimated. The theoretical potential improvement in SNR of SASB over DRF has been estimated based on the measured SNR data and an assumption of a stationary image object, and a correct phase-alignment in the 2nd stage beamformer. The improvement is attained at the entire range and at a depth of $80 \mathrm{~mm}$ the improvement is $8 \mathrm{~dB}$.

\section{References}

[1] L.J. Cutrona, W.E. Vivian, E.N. Leith, G.O. Hall, A high resolution radar combatsurveillance system, IRE Trans. Mil. Elect. MIL-5 (1961) 127-131.

[2] C.W. Sherwin, J.P. Ruina, D. Rawcliffe, Some early developments in synthetic aperture radar systems, IRE Trans. Mil. Elect. MIL-6 (1962) 111-115.

[3] J.C. Curlander, R.N. McDonough, Synthetic Aperture Radar: Systems and Signal Processing, John Wiley \& Sons Inc., 1991.

[4] M. Karaman, P.C. Li, M. O’Donnell, Synthetic aperture imaging for small scale systems, IEEE Trans. Ultrason. Ferroelec. Freq. Contr. 42 (1995) 429-442.

[5] R.Y. Chiao, L.J. Thomas, S.D. Silverstein, Sparse array imaging with spatiallyencoded transmits, in: Proc. IEEE Ultrason. Symp. 1997, pp. 1679-1682.

[6] J.T. Ylitalo, H. Ermert, Ultrasound synthetic aperture imaging: monostatic approach, IEEE Trans. Ultrason. Ferroelec. Freq. Contr. 41 (1994) 333-339.
[7] M.H. Pedersen, K.L. Gammelmark, J.A. Jensen, In-vivo evaluation of convex array synthetic aperture imaging, Ultrasound Med. Biol. 33 (2007) 3747.

[8] D.K. Peterson, G.S. Kino, Real-time digital image reconstruction: a description of imaging hardware and an analysis of quantization errors, IEEE Trans. Son. Ultrason. 31 (1984) 337-351.

[9] G.R. Lockwood, J.R. Talman, S.S. Brunke, Real-time 3-D ultrasound imaging using sparse synthetic aperture beamforming, IEEE Trans. Ultrason. Ferroelec. Freq. Contr. 45 (1998) 980-988.

[10] C.R. Hazard, G.R. Lockwood, Theoretical assessment of a synthetic aperture beamformer for real-time 3-D imaging, IEEE Trans. Ultrason. Ferroelec. Freq. Contr. 46 (1999) 972-980.

[11] M. O’Donnell, L.J. Thomas, Efficient synthetic aperture imaging from a circular aperture with possible application to catheter-based imaging, IEEE Trans. Ultrason. Ferroelec. Freq. Contr. 39 (1992) 366-380.

[12] C. Passmann, H. Ermert, A $100-\mathrm{MHz}$ ultrasound imaging system for dermatologic and ophthalmologic diagnostics, IEEE Trans. Ultrason. Ferroelec. Freq. Contr. 43 (1996) 545-552.

[13] C.H. Frazier, W.D. O'Brien, Synthetic aperture techniques with a virtual source element, IEEE Trans. Ultrason. Ferroelec. Freq. Contr. 45 (1998) 196-207.

[14] S.I. Nikolov, J.A. Jensen, Virtual ultrasound sources in high-resolution ultrasound imaging, in: Proc. SPIE - Progress in biomedical optics and imaging, vol. 3, 2002, pp. 395-405.

[15] S.I. Nikolov, J.A. Jensen, 3D synthetic aperture imaging using a virtual source element in the elevation plane, in: Proc. IEEE Ultrason. Symp. vol. 2, 2000, pp. 1743-1747.

[16] M.H. Bae, M.K. Jeong, A study of synthetic-aperture imaging with virtual source elements in B-mode ultrasound imaging systems, IEEE Trans. Ultrason. Ferroelec. Freq. Contr. 47 (2000) 1510-1519.

[17] J. Kortbek, J.A. Jensen, K.L. Gammelmark, Synthetic aperture focusing applied to imaging using a rotating single element transducer, in: Proc. IEEE Ultrason. Symp. 2007, pp. 1504-1507.

[18] J.A. Jensen, N.B. Svendsen, Calculation of pressure fields from arbitrarily shaped, apodized, and excited ultrasound transducers, IEEE Trans. Ultrason. Ferroelec. Freq. Contr. 39 (1992) 262-267.

[19] J.A. Jensen, Field: a program for simulating ultrasound systems, Med. Biol. Eng. Comp. 10th Nordic-Baltic Conference on Biomedical Imaging 4(Supplement 1, Part 1) (1996b) 351-353.

[20] J. Kortbek, S.I. Nikolov, J.A. Jensen, Effective and versatile software beamformation toolbox, in: Proc. SPIE - Medical Imaging - Ultrasonic Imaging and Signal Processsing, vol. 651319, 2007, pp. 1-10.

[21] N. Oddershede, J.A. Jensen, Effects influencing focusing in synthetic aperture vector flow imaging, IEEE Trans. Ultrason. Ferroelec. Freq. Contr. 54 (2007) $1811-1825$

[22] L.F. Nock, G.E. Trahey, Synthetic receive aperture imaging with phase correction for motion and for tissue inhomogenities - Part I: basic principles, IEEE Trans. Ultrason. Ferroelec. Freq. Contr. 39 (1992) 489-495.

[23] G.E. Trahey, L.F. Nock, Synthetic receive aperture imaging with phase correction for motion and for tissue inhomogenities - Part II: effects of and correction for motion, IEEE Trans. Ultrason. Ferroelec. Freq. Contr. 39 (1992) 496-501.

[24] H.Ş. Bilge, M. Karaman, M. O'Donnell, Motion estimation using common spatial frequencies in synthetic aperture imaging, in: Proc. IEEE Ultrason. Symp. 1996, pp. 1551-1554.

[25] M. Karaman, H.Ş. Bilge, M. O'Donnell, Adaptive multi-element synthetic aperture imaging with motion and phase aberration correction, IEEE Trans. Ultrason. Ferroelec. Freq. Contr. 42 (1998) 1077-1087.

[26] C.R. Hazard, G.R. Lockwood, Effects of motion artifacts on a synthetic aperture beamformer for real-time 3D ultrasound, in: Proc. IEEE Ultrason. Symp. 1999, pp. 1221-1224.

[27] J.S. Jeong, J.S. Hwang, M.H. Bae, T.K. Song, Effects and limitations of motion compensation in synthetic aperture techniques, in: Proc. IEEE Ultrason. Symp. 2000 , pp. $1759-1762$.

[28] S.I. Nikolov, J.A. Jensen, K-space model of motion artifacts in synthetic transmit aperture ultrasound imaging, in: Proc. IEEE Ultrason. Symp. 2003, pp. 18241828.

[29] K.L. Gammelmark, J.A. Jensen, Multielement synthetic transmit aperture imaging using temporal encoding, IEEE Trans. Med. Imag. 22 (2003) 552563.

[30] K.L. Gammelmark, J.A. Jensen, Duplex synthetic aperture imaging with tissue motion compensation, in: Proc. IEEE Ultrason. Symp. 2003, pp. 1569-1573.

[31] S.I. Nikolov, K. Gammelmark, J.A. Jensen, Recursive ultrasound imaging, in: Proc. IEEE Ultrason. Symp. vol. 2, 1999, pp. 1621-1625. 